Review

\title{
Presents Some Biologically Structured Materials
}

\author{
${ }^{1}$ Relly Victoria Virgil Petrescu, ${ }^{2}$ Raffaella Aversa, ${ }^{2}$ Antonio Apicella and ${ }^{1}$ Florian Ion Tiberiu Petrescu

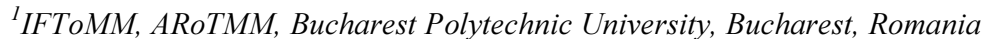 \\ ${ }^{2}$ Advanced Materials Lab, Department of Architecture and Industrial Design, Second University of Naples, Aversa, Italy
}

Article history

Received: 16-11-2019

Revised: 25-01-2020

Accepted: 27-01-2020

Corresponding Author:

Florian Ion Tiberiu Petrescu

Bucharest Polytechnic

University, Bucharest,

Romania

Email: fitpetrescu@gmail.com

\begin{abstract}
In this paper bio-tissue, mathematical modeling serves as a central repository to interface design, simulation and tissue fabrication. Finite element computer analyses will be used to study the role of local tissue mechanics on endochondral ossification patterns, skeletal morphology and mandible thickness distributions using single and multi-phase continuum material representations of clinical cases of patients implanted with the traditional protocols. New protocols will be hypothesized for the use of the new biologically techno-structured hybrid materials.
\end{abstract}

Keywords: Stem Cells, Biomaterials, Biologically Structured Materials, Techno-Structured Materials, Hybrid Materials, Biotechnology, Bioengineering, Bioactive Scaffolds, Biomimetics, Endochondral Ossification Patterns, Finite Element Analysis, Osteointegration, Osteoinduction, Skeletal Morphology, Tissue Mechanics

\section{Introduction}

Whenever we think of an organ of the human body we should look at it as an active component of the whole organism and not just as a separate organ. All matter has a certain life in the sense that it is born in a certain form and then it ages, undergoing permanent transformations that lead to its aging and changes of state, form and appearance. Organic living matter, unlike inorganic matter, is not composed of atomic chains that form molecules but is based on the living cell, the cell which is composed basically of atoms and molecules, including molecular chains, so unlike inorganic matter which is on essence structured from atoms and molecules, organic living matter is structured from living cells, which fulfill the fundamental roles of life as we know it today on our blue planet.

It is a great lack to look at a living organ belonging to an organism only from the anatomical point of view of the respective organ because each organ has several functionalities that it also performs in correlation with the other organs of the whole human body.

For example, the bone system has not only the apparently main function of supporting and defending the whole human body. Perhaps this role of dressing, supporting and protecting all the other organs is a basic one, but not the most essential one fulfilled by the bone system. The bone system has many roles to play in the human body, but its most important role is to support the circulatory system by creating fresh blood permanently even within the spinal cord, but also from young or adult bones, through the creation of all cells of the blood, of which there are mainly four types of cells, namely, the red cells that have the role of transporting and nourishing the whole body with oxygen, thus carrying out the oxygenation function of the whole organism up to the level of the cells, then the white cells of different types which have different roles in defending the body from various dangerous intruders, thirdly it is the platelet cells that will stop the bleeding that can start with the various wounds that are created in the life of the body and fourthly it is the stem cells essential for a rapid restoration of any type of cells depending on the organ that is affected. In general, stem cells that can also differentiate when they reach a particular organ are also produced within the bone marrow and are also transported by blood directly where it is necessary to repair a tissue belonging to a particular injured organ. For this reason, the human body is provided with a certain symbiosis between the emergency stem cell repair cells and the platelet cells that stop the rapid accidental bleeding produced in the body. This symbiosis occurs since their birth and for this reason, they are embedded together within placentae so that when circulating through the blood, they will be placentally coupled to both platelet and stem cells, being ready to act together as soon as possible an emergency injury, or another extreme situation occurs within a point of the entire body. Platelet cells from the placental masses that enclose and transport them immediately stop 
bleeding to a wound produced and the stem cells immediately enter into action and begin tissue reconstruction at the affected site no matter what type of tissue or cells would be where the injury occurred because the initial stem cells created in the bone marrow are capable of developing and transforming into any type of human cell, adapting rapidly to any area or point of the human body, a huge advantage that these cells have compared to other cells that multiply in their workplace and by living permanently restoring the organ of which they belong, but which could not adapt to any other organ as the wonderful stem cells can do. So until now, four such basic products of bone marrow are known, as we have just shown, these are the main components of human blood, without which the blood that is restored permanently and regenerates in the bone marrow (so this fresh blood), would not it could exist and at that moment the body could no longer live.

Going back to the stem cells, it should be pointed out that they move through the blood within the body and if we want to collect them more easily so that they can be quickly injected into a certain place in the body in order to repair that area that was previously affected by something, then the easiest way to collect stem cells is to separate them together with platelets directly from the blood.

At this point, it is necessary to pause a little to highlight another essential role of the bone system within the body. The human body begins to age when it no longer produces enough fresh blood because of the lack of bone marrow that over time begins to become more less. The bone system together with the liver, lungs and kidneys, represents the main reservoir of water, minerals and blood, as well as the main storehouse of water, minerals and blood and thus indirectly and the main energy reservoir of the body. Specialized micron small cells, called mitochondria, have the primary role of producing energy for the body by creating energy ATP molecular chains, from water and air plus phosphorus, extracted from water and air from the body and from phosphorus from the bone reservoir, but then when the bone reservoir begins to lose from water and phosphorus reserves and the amount of energy raw material available to the mitochondria in the body decreases, leading to a decrease in the raw materials available for mitochondria and thus the energy production at the cellular level decreases and with it and the production of mitochondria and healthy new cells. Here it seems to be a vicious circle that is hard to stop, but in reality, the fault is genetically exclusive, because the bone system is genetically programmed to age, at one point the bones never can further produce marrow and blood and then only the ends of the bones help the spinal cord to produce the marrow and blood and at some point, they will not be able to do so and all the production of marrow and blood then remains in the spinal cord. If other organs can be permanently restored especially in the presence of fresh blood, the bones are sadly destined to age in several stages, genetically programmed. Although there is a large reserve of water and blood in the liver, kidneys and lungs, the basic reserve is that of bone, which has in its component water, minerals, phosphorus, blood, fat, able to stick and build the cell glue that is used in the cells but also between them, uniting the living cells to hold them together and in addition, only the bone reservoir can build the marrow of bone, spinal cord and blood, but also energy, basic energetic substances, to be donated to the mitochondria that will it then produces ATPs (energy molecular chains). If the bone system gets old and deteriorates over time, it is obvious that then the blood and energy will decrease and the water supply in the body will decrease and, at birth, it is $90 \%$, in a child, it becomes $80 \%$, in adolescents of about $70 \%$, in adults, it drops to $65 \%$ and in older people, it reaches $50 \%$ and below. Below $45 \%$ the water supply can no longer support the recovery of the blood in the required amount, nor of the ATP energy and the aging body will suffer from lack of energy, lack of oxygenation, lack of water and food, but also lack of extraction in time of normal toxins returned from the cells to the kidneys and liver also through the blood.

At least from this point of view, the restoration of the broken or accidentally broken bones should be done in such a way that it does not affect the bone tissues and their life and functions, so as not to prematurely age the human bone system, an essential system for sustaining the life and vitality of the body.

Bone regeneration of bone defects is still a major clinical challenge in maxillofacial and orthopedic surgery. LCP without stem cells or growth factors is used today. LCP prevents soft tissue from entering the defective space and is biocompatible with bone. It is also mechanically stable and can, therefore, maintain the bioreactor's shape and promote intramembranous ossification. A new bioreactor system is based on previous reports that biphasic ES increased proliferation of human stem cells, such as a human Mesenchymal Stromal Cell (hMSC) and several growth factors, such as Vascular Endothelial Growth Factor (VEGF), BMP-2 and interleukin -8 (IL-8) (Kim et al., 2011; 2013). These properties offer great potential to create favorable microenvironments for bone regeneration. VEGF is a critical factor for angiogenesis and IL-8 is a chemokine for stem cell recruitment.

It is known that the effect of two-phase electric current on long and thin cells, such as MSC, is proportional to the spatial derivative of the electric field (Lee et al., 2016; Rattay, 1989), which is large near the electrode surface. The increased VEGF production of cells near the electrode leads to a gradient of VEGF 
towards the electrode, encouraging angiogenesis in the direction of the gradient (Gerber et al., 1999). An ACS sheet has a porous structure that supports the proliferation and differentiation of bone cells with a higher cellular affinity (Oliveira et al., 2010). The molecular structure of ACS has an electrical polarity that holds rhBMP-2 on the surface of ACS with electrostatic force (Geiger et al., 2003). An in vivo bioreactor system with ES benefits from a direct one-step application compared to a two-step procedure, in which the bioreactor is transplanted first to the well-vascularized heterotopic sites and then the regenerated tissue is transplanted to the orthotopic site. (Wiltfang et al., 2016).

It should be noted that the calcium/calmodulin complex binds and activates enzymes such as calcium/calmodulin-dependent protein kinase (CaMK) (Braun and Schulman, 1995), Calcineurin (Cn) (Winslow et al., 2006) and Nitric Oxide Synthase (NOS) (Stuehr and Haque, 2019).

CAMK enhances cell proliferation by activating the cAMP Response Element-Binding protein (CREB) (Pinto et al., 2015; Wheeler et al., 2008) and CyclinDependent Kinase (CDK) with cyclin (Kahl and Means, 2003). Cn activates the Nuclear Factor of Activated $T$ cells (NFAT) and transfers the transcription factor to the nucleus (Hogan et al., 2003). Although NFAT is associated with osteoclast activation and early bone resorption (Kim et al., 2018), NFAT enhances osteoblast proliferation during fracture repair (Adamopoulos, 2018). NOS promotes cell proliferation by increasing nitric oxide levels.

Regeneration of large bone defects has been a challenging issue in maxillofacial and orthopedic surgery. Although there are efficient and wellestablished treatments such as distraction osteogenesis and autologous bone grafting, all methods have limitations that are being tackled by many kinds of research (Dimitriou et al., 2011). Distraction osteogenesis can achieve bone regeneration by moving existing bone at the speed of osteogenesis in the stretched callus, where the time needed for the treatment is proportional to the defect size (Glowacki, 1998). Autologous bone grafting being current gold standard can achieve bone union regardless of the defect size with advanced surgical techniques (Azi et al., 2016); however, donor site morbidity is inevitable after the harvesting of the graft. Other bone regeneration treatments with osteoconductive cell-free substances such as allogenic, xenogenic or alloplastic bone grafts showed low osteogenesis efficacy with long regeneration times (Lim et al., 2018). Bone regeneration with a growth factor such as recombinant human Bone Morphogenetic Protein (rhBMP)-2 showed enhanced bone quantity with reduced regeneration time in the clinical application (Ha et al., 2017; Kim et al., 2016; Urist and Strates, 1971).
Bone Morphogenetic Protein (BMP) is a known osteoinductive signaling chemokine, which determines bone formation by recruiting stem cells and differentiating them into bone-forming cells (Kim et al., 2010).

Among the BMP family, rhBMP-2 is widely used for bone tissue engineering due to its bone formation efficiency and commercial accessibility. Despite its efficacy, rhBMP-2 is rapidly diffused when injected in vivo, therefore a large dose is often required to achieve sufficient osteoinductive effect (Song et al., 2016). However, a high dose of rhBMP is associated with swelling of soft tissue hematoma, radiculopathy, cystlike bone formation, resorption and bone inflammation (Cho et al., 2017; Mesfin et al., 2013; Shields et al., 2006; Zara et al., 2011). Moreover, cell proliferation is not stimulated by rhBMP-2, while numerous cells are required for bone regeneration at large bone defects.

Direct implantation of precultured stem cells is a simple and efficient method (Yoon et al., 2018). However, applying stem cells for bone regeneration requires multiple and complicated procedures, such as tissue harvesting, stem cell separation and cell proliferation and differentiation, which are accompanied by long periods of culture, high costs and the risk of infection (Bueno and Glowacki, 2009). Moreover, the efficiency of stem cell transplantation is low due to the low survival rate after transplantation, especially in large bone defects due to insufficient angiogenesis (Ankrum and Karp, 2010).

Biomimetics, biomechanics and tissue engineering are three multidisciplinary areas that have been considered in this research to achieve the goal of improving the reliability of prosthetic implants. As test methods and mathematical methods are closely linked, a promising approach seems to be the combination of in vitro and in vivo experiments with computer simulations (in silico). An innovative approach for biomimetic and biomechanics is presented and a new synthetic structure that provides a micro-environment, mechanical and nutritional in accordance with the cultures of tissue osteoblast cells used in regenerative medicine.

The new hybrid ceramic-polymer nanocomposites are mutually investigated by the biomimetic modeling of Finite Element Analysis (FEA), anatomical reconstruction, quantitative tomographic characterization, tissue scaffold calculation. Starting materials are a class of innovative high-quality bio-polymeric materials, highquality hybrids, constituted by the proposed research group, which will be used as a bioactive matrix for the preparation of bio-mineralized nanocomposites.

This study treats biomimetics, biomechanics and tissue engineering as highly correlated multidisciplinary areas, combined with the design of bone tissue scaffolds. Bone growth, maintenance and ossification are fundamental and are regulated by mechanical indices imposed by physical activities: This biomimetic/biomechanical approach will 
be followed by the design of in vitro mineralization procedures and the ossification of the scaffold. Mathematical modeling of bio tissues serves as a central repository for interface design, simulation and fabrication.

Finite element computer analyses will be used to study the role of local tissue mechanics on endochondral ossification patterns, skeletal morphology and mandible thickness distributions using single and multi-phase continuum material representations of clinical cases of patients implanted with the traditional protocols. New protocols will be hypothesized for the use of the new biologically techno-structured hybrid materials.

It is known that the field of interdisciplinary research of materials for biomedical applications is structured and calibrated today in the bone repair study, which is basically its basis.

The bones are considered to be a biological hybrid material composed of an organic component, collagen and an inorganic nanocrystalline hydroxyapatite component. Both phases integrate on a nanoscale so that morphological and physical variables such as crystal size, nano-orientation, short-order order between the two components determine the characteristics of its nanostructure and therefore the mechanical properties of the different types of bone (Frost, 1964; 1990; 2003).

Based on bone regeneration criteria, new bioactive biomaterials have been developed. These materials are expected to favor the formation of bone tissue by stimulating the proliferation and differentiation of osteoblasts (Schiraldi et al., 2004; Aversa et al., 2016a; 2016b; 2016c; 2016d; 2017a; 2017b; 2019; Syed et al., 2017).

The use of nanostructured materials similar to natural bone tissue is one of the most promising options for bone healing. Nanotechnologies for the implementation of organo-inorganic hybrid materials offer excellent chances to improve the performance of existing conventional bone implants (Aversa et al., 2016a; 2017).

Current research evaluates the evolution of polymer-polymer hybrids for bone tissue repair, as well as chemical procedures that control the nanostructuring of materials.

The objective of the paper is inherent in the following scientific fields:

- biomechanics and human biophysical bone

- Biomimetics: Nanotechnology in medicine for materials inspired by nature

- Bioactive scaffolds favoring osteointegration in nanocomposite and hybrid porous structural matrices

\section{Biofidelity Advances}

Recent studies of the mandible (Schwartz-Dabney and Dechow 2003; Apicella et al., 2010; Aversa et al., 2016a; 2016b) and FEM modeling of teeth (Sorrentino et al., 2007; Apicella et al., 2010).

Knowing the mechanical and adaptive features of the bone is an essential issue in designing new biomimetic prostheses to replace a bone with minimal biological and biomechanical invasiveness.

Biomimetics is the science that investigates such aspects and can be considered the natural junction between biology and engineering. This competence convergence allows the development of biological principles and models to produce bio-inspired materials that can be used to fully design prosthetic tissues and systems.

New generations of concepts could be generated by the conscious investigation of biomimetics, which can provide clinical tools to restore the structural, biomechanical and aesthetic integrity of bone functions.

Recent advances in cellular and molecular biology and material engineering science (nanotechnology) have established that biomimetics and tissue engineering are developed to improve the integral integration of prosthetic and restorative implants (Aversa et al., 2016a; 2016b; 2016c; 2016d; Perillo et al., 2010; Annunziata et al., 2006; 2008).

Since last century, parts of our body have been replaced with artificial prostheses. The materials used for these devices were chosen not to produce adverse responses in contact with human body tissues and physiological fluids.

The criteria for choosing a specific biomaterial were related to its biocompatibility and functionality, which could be directly associated with interfacial bone/implant interactions at a nanometric level. Only in the 1990s, the study of these interference effects was improved by the use of thin nanometer layers and surface changes.

It then generated a great commercial interest in the orthopedic market of adopting new modified implants of nanoparticles that promote soft and soft tissue engineering.

\section{New Classes of Biomaterials}

There are several ways in which living tissues can react to synthetic implant materials, but are essentially limited to their response to the interface material

Three main terms could describe the behavior of biomaterials defined by Jones and Clare (2012), Hutmacher (2000) and Hoppe et al. (2011).

Namely, tissue responses are divided into:

\section{- Bioinert}

- Bioresorbable

- Bioactive

A further classification of ceramic biomaterials can be made according to their reactivity to physiological fluids: 
- Bioinert, such as Alumina for dental application

- Bioactive, such as hydroxyapatite used as a coating on metallic implants

- The active surface, such as bio-glass or A-W bottles

- Bio-resorbed, such as tri-calcium phosphate

Further improvement of these properties can be accomplished using nanostructured bioceramics that can be used as interactive materials, helping natural tissues heal by promoting tissue regeneration and restoring physiological functions (Schiraldi et al., 2004; Mano et al., 2004; Morales-Hernandez et al., 2012; Mouriño et al., 2012).

This approach has been studied in this study to develop a new generation of nanostructured bio-ceramicpolymeric hybrids that can be used in a wider range of medical applications (Petrescu and Calautit, 2016a; 2016b; Petrescu et al., 2015; 2016a; 2016b; 2016c; 2016d; Petrescu and Petrescu, 2017; 2018).

Porosity is one of the keys to the success of these materials and is increasingly adopted when a narrowing of the bones is required and strong implant stability.

\section{Tissue Engineering New Perspectives}

For several years, tissue engineering has benefited from the combined use of live stem cell intake in threedimensional ceramic scaffolds. This strategy is completed to provide healthy cells directly to the damaged site (Bonfield et al., 1981; Hench and Wilson, 1993; Hench and Polak, 2002; Hench and Thompson, 2010).

By combining the traditional bio-ceramic implant with the already assimilated knowledge of stem cell growth and differentiation in clinically and productively developed osteogenic strategies.

Stem cells developed in ceramic nanobiocomposites should be adopted in the case of extensive bone repair with excellent prospects of good functional recovery and integration of the hybrid scaffold with bone.

Synthetic Hydroxyapatite (HAp) has been described in the literature as an attractive material for bone implants (Kim et al., 2004; Morales-Hernandez et al., 2012).

Since its adoption, the most common and simplest method of producing synthetic HAp is the solid reaction between calcium and phosphate ions, resulting in the formation of powdered compounds which can be sintered and recovered at elevated temperature to form a compact polycrystalline structure Julien et al. (2007).

The HAp bioactivity is governed by processing parameters, such as the initial compounds, the size of the crystalline granules, their purity and the ratio of calcium to phosphorus atoms. In particular, nanocrystals have shown improved bioactivity due to their large surface area. The use of hydroxyapatite with nanoparticles has been proposed as a valid solution for the consolidation of low strength polymer scaffolds.
The use of HAp nanoparticles for new classes of implants, biocompatible coatings and high strength nanocomposites can be developed (Gorustovich et al., 2010).

\section{Biomimetics}

A general feature of several tough natural hybrid materials, such as bones, sea urine teeth, pearls, is the strong nanometric interaction between inorganic and organic phases.

This feature allows the organic phase to act as a nanometric scale as a plastic energy dissipation network that inhibits crack propagation (high resistance); s, in situ synthesis techniques, have been adopted to mimic naturally occurring processes. In particular, precipitation of hydroxyapatite (or other crystalline compounds) in a polymeric matrix was considered a viable route to produce biomimetic composites.

\section{Organic-Inorganic Hybrid Biomaterials}

A bioinspired material development approach considering the formation of self-assembling hybrid organic-inorganic will favour the use of hybrids in biomedical applications. The high versatility of these hybrids offers main functional and structural advantages that lead to the possibility to tailor-design materials in terms of shape and chemical and physical properties.

\section{Bioengineering and Bioactive Scaffolds}

For nanotechnology nanomaterials, nanotechnology is increasingly adopted for emerging applications such as coating systems or three-dimensional coating systems (tecto) (Aversa et al 2016a; Karageorgiou and Kaplan, 2005; Sorrentino et al., 2007). Critical, micro and nanotechnologies show the potential to be used to produce advanced models for fundamental studies, such as tissue engineering structures or bio-molecular devices.

The ideal material for bone skeletons has always been a hot topic for research. An ideal skeleton should provide a sufficiently rigid but durable mesh to temporarily replace the damaged bone. At the same time, it should be able to biodegrade after the formation of new tissues and integrate it fully (Montheard et al., 1992; Kabra et al., 1991; Peluso et al., 1997; Schiraldi et al., 2004).

Amorphous nanoparticles were synthesized in the laboratory. A new class of polymer-ceramic hybrid materials simulating the mechanical behavior of the bone were used as potential candidates for scaffolding (Syed et al., 2017).

The result of these self-assembled nanostructured composites was micro-foamed and tested as a new preimplantation skeleton that can accommodate osteoblast growth factors or stem cells to differentiate osteoblasts. 


\section{Biofidelity Models and FEM Analysis}

Understanding the biological mechanisms of healthy and healthy bone growth is an iterative process between biology and engineering. During this process, knowing that reverse engineering of a biological system can have positive feedback in the field of biology, allowing for a more complete and secure understanding of the potential path for further development of applied medical engineering.

The most important question is how clinician interference with biological systems can be optimized to improve treatment options so as to increase treatment efficiency and lead to a more stable outcome.

The use of new diagnostic and engineering tools such as those used in our research (for example, CT and CTM $\mathrm{CT}$ segmentation and solid CAD reconstruction) can detail the anatomy of soft and soft textures in a very precise manner as a small standard deviation. Therefore, the integration of biological knowledge and clinical possibilities is essential. A more reliable and biophysical model begins with biomechanical modeling of bones, ligaments and alveolar bone, using Finite Element Analysis to gain insight into the biological response to changing biomechanical circumstances.

Because current tests and numerical methods are closely related, a methodological approach is to combine in vitro and in vivo experiments with computer simulations (in silico). There are, however, a number of stimulus points involved in creating and realizing the mathematical model. The concurrent interaction of several variables that influence the prosthetic system was investigated by simulation in the mathematical modeling of finite elements.

Finite Element Analysis (FEA) involves the subdivision of a geometric model into a finite number of elements, each with specific mechanical properties. The variables to be investigated are guessed with mathematical functions. Specific mathematical software evaluates the distribution of stresses and stresses in response to changing charging conditions.

A complete assessment of the mechanical behavior of a solid or prominent biological structure can be made, even in the case of non-homogeneous organisms. When properly validated by in vivo or in vitro testing, finite element analysis is useful in defining optimal recovery criteria and material selection criteria while allowing for potential fracture prognosis in limited circumstances.

\section{Materials and Methods}

\section{Materials}

$300 \mathrm{~m}^{2} \mathrm{~g}^{-1}$ hydrophilic matrix for amorphous silica filling (Aerosil 300 Degussa, Germany) with a specific surface area of 2 n-hydroxyethyl methacrylates (HEMA),
Sigma-Aldrich Chemicals Co. was used. The initiator of the radical polymerization reactions used as azobisisobutyronitrile (AIBN) obtained from Fluka (Milan, Italy). HEMA monomers were mixed with $10 \%$ by volume of silica. The degreased resin was cast in 2.5 $\mathrm{mm}$ thick flat shapes and polymerized at room temperature, controlled at $60^{\circ} \mathrm{C}$ for $24 \mathrm{~h}$. Another one hour end at $90^{\circ} \mathrm{C}$ was finally obtained on the nanocomposite plates.

The microporosity was induced in the dense material, first balancing the samples in pure ethyl alcohol and then rapidly extracting the absorbed alcohol, balancing it in distilled water. During the rapid extraction and counterdiffusion of alcohol, there is a microporosity in the ceramic-polymer hybrid material, evidenced by the intense bleaching of the treated system.

\section{Finite Element Analysis (FEA)}

Segmentation of medical images was derived from CT using Mimics software (Materialize, Belgium) to process a medical image to the patient. As reported at the top of Fig. 1, CT processing resulted in a solid 3D solid model of bone anatomy and structure.

The combined use of Mimics and 3-Matic software (Materialize, Belgium) was used to obtain solid 3D models and Finite Element Analysis (FEA).

External bone geometry was reconstructed by generating a three-dimensional volume that interpolates CT scans. The results were then imported into 3Matic software for surface and solid optimization, finite element modeling and material properties of different bone sections depending on literature characteristics (Beaupre and Hayes 1985; Reilly and Burnstain 1974; 1975; Huiskes et al., 1987; Schwartz-Dabney and Dechow, 2003, Töyräsa et al., 2001).

Bone stress can be modulated by choosing skeletal swollen thickness for healthy bone growth. In vivo tests using these modified oral implants confirmed the improved ability of these implants to promote early osteointegration (Gramanzini et al., 2016).

Biomimetic/biomechanical approach: Hybrid ceramic-polymer design and bulk design to improve osteointegration.

Bioprotective devices are large-scale rehabilitation therapy in clinical practice in many areas of rehabilitation surgery, such as dentistry, maxillofacial surgery, orthopedics. The bone and implant interface has been studied for several years as we try to move from bioinert to bioactive biomaterials. In fact, the histological analysis carried out in these years does not allow the confirmation of possible theories of contact, junction systems and others. However, there is fluid contact between the osteocyte canal and the implant surface. Bioactive biomaterials could favor and amplify the differentiation of an osteoblastic phenotype that occurs during the healing of 
surgical wounds caused by the implant, with better osteointegration in a shorter time. Recent studies describe the characteristics of nanostructured materials that could promote osteointegration:

- Carbon and alumina nanostructures, which mimic the nano-dimensional geometry of hydroxyapatite, increase osteoblastic activity and thus produce larger bone deposits when applied to orthopedic implants.

- nanostructured biomaterials that mimic the bioactivity of hydroxyapatite crystals favor the adhesion and production of alkaline phosphates in osteoblast-like cells

Therefore, further studies on these materials could lead to better and shorter healing to promote protocols to ensure early and immediate loading. The composition and properties of the surface appear to be important, as they seem to modulate the response of osteoblastic cells that affect tissue healing (Davis et al., 1991; Gramanzini et al., 2016; Aversa et al., 2016b).

The implanted tissue adapts its composition and architecture to its functional load (Apicella et al., 2011; 2015). Therefore, a key to the success of the titanium implant to integrate into the bone appears to be whether or not bone remodeling is correct at the periphery of the implant (Aversa et al., 2016b).

Figure 1 shows the result of the "in vivo" experiments performed on dental implants placed in the femur of the white rabbit. In particular, the experiment described in
Aversa et al. (2016b) consisted of the evaluation of the osteoinductivity and osteoconductivity of the Ti implant surfaces without a 100 microns thin layer hybrid material from ceramic material.

Bone implantation or bone thickening, which is defined as the percentage of bone implants for biomimetically implanted implants that are not covered by the six-month in vivo test, shows a significant improvement of approximately $100 \%$ growth over two months. and $30 \%$ after 6 months.

The reconstruction of the micro-CT bone around the implant was validated using the physiological distributions of the calculated FEA strains. The maps of the maps around the bone around the implant confirmed the critical role of the Ti-Bone bioactive interface.

The proliferation of osteoblast and bone growth in the implanted rabbit femur are clearly favored and accelerated by the presence of the hybrid nanostructure layer.

The biomechanical approach using adaptive bone properties describes the biomimetic behavior of the proposed hybrid scaffold prior to implantation, as it can predict bone resorption surfaces (elements from the FEA model with lower physiological limits have been removed in the image), as shown in the in vivo microtubule implant micro-reconstruction (on the right side of Fig. 1).

Research has shown that mechanical stimulation can have a profound effect on the differentiation and development of mesenchymal tissues.

Figure 2 illustrates the adaptive properties and strain threshold values for healthy bone growth.

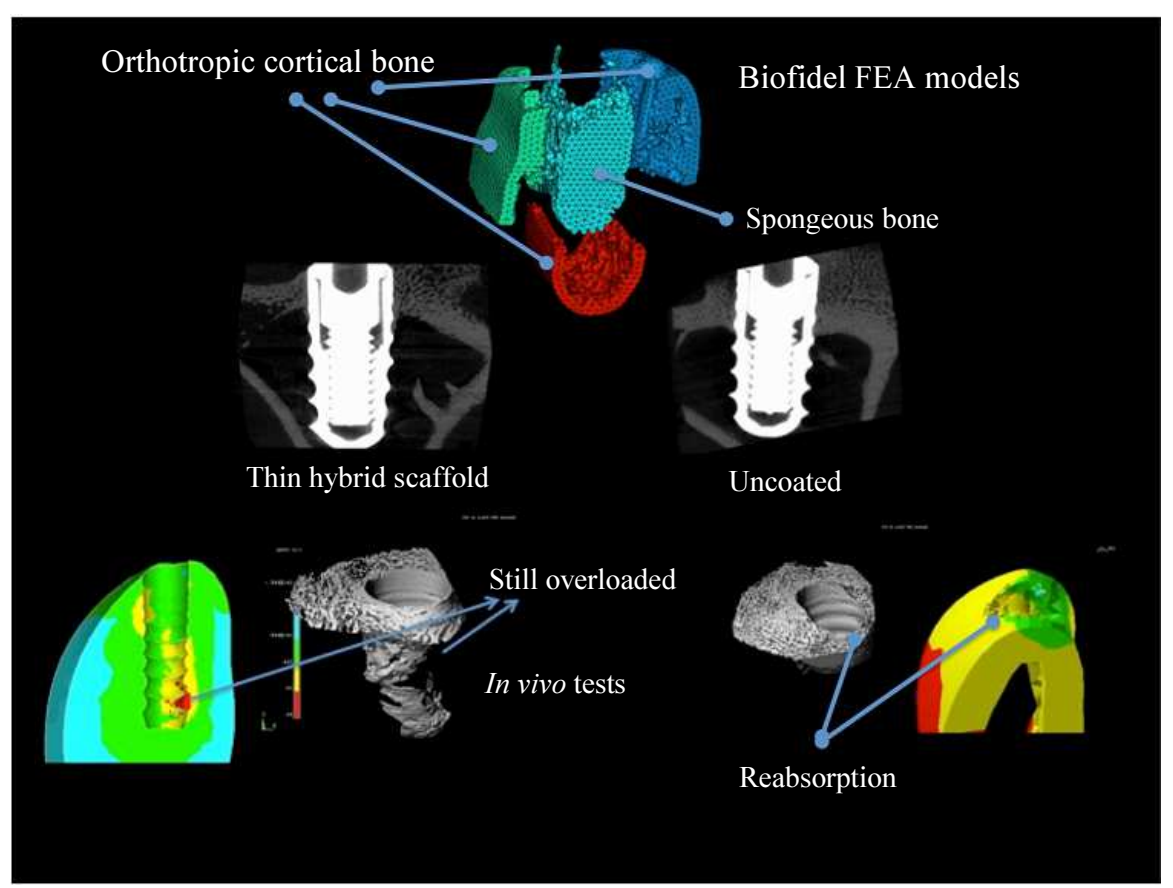

Fig. 1: In silico and in vivo validation for Osteoconduction of Titanium implants coated with a nanostructured hybrid osteoactive (left side) and without (right) 


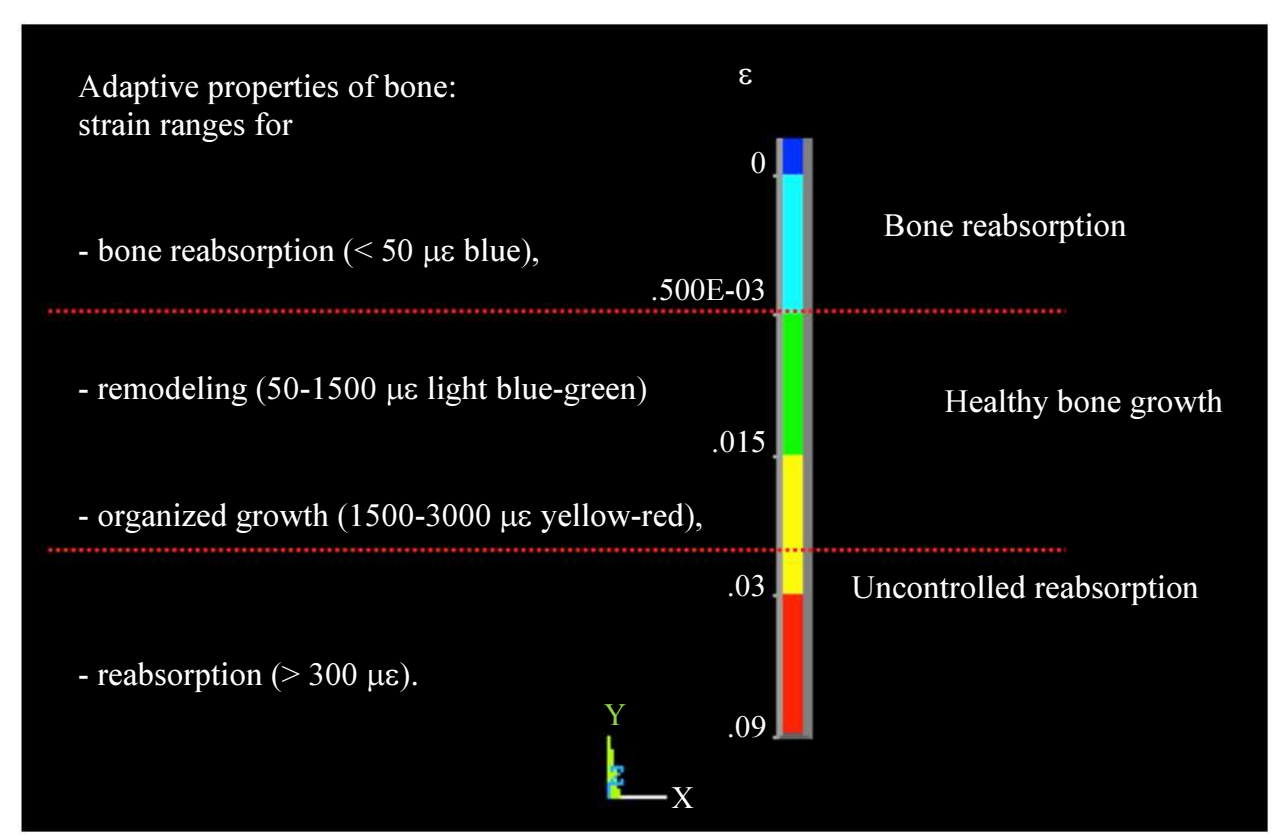

Fig. 2: Frost (1990) adaptive window of bone physiology: Structural adaptations to mechanical usage

According to Frost (1990), which quantified Wolff (1892) above (> 3000 micro epsilon) and below ( $<50$ micro epsilon) of critical strain levels, bone growth is impaired. In the mild strain region, healthy bone growth and regeneration is favored. In fact, in order to maintain implant stability during pregnancy, it is of major importance for bone-forming osteoblasts to promote an extracellular matrix in the immediate vicinity of the implant. Osteointegration mechanisms to be considered in biofidel models.

Osteointegration of implants is essential for the rehabilitation of the prosthesis. Achieving and maintaining stable functional anchylosis has the following morpho-structural characteristics, namely:

- Direct contact between bone and implant in the absence of the idoneous tissue interface

- The existence of the primary bone in contact with the surface of the biomaterial

- Deposition, externally to the primary bone, of the lamellar secondary bone in contact with the titanium surface

- Increase in preimplantation bone density compared to the normal bone region of the region

- Increase in medullary spaces, which is required to evacuate the metabolic requirements of less involved tissues in the region in the dissipation of the load

- Compact bone condensation, which may be related to the load propagation patterns determined by the specific morphology of the implant

- Organizing a strong trabecular structure that is radially removed from the compact preimplantation bone
- The presence of a bone crystal wall at the level of the subepithelial conjunctiva, which may allow junctional tropism and the formation of the liquid epithelium

The mature matrix, which has been described to occur in dental and orthopedic clinical trials, is expected to provide mechanical stability of the implant even in the early osseointegration phase (primary stability). In fact, due to the hydrophilic nature of the hybrid material, high fluid levels are absorbed from the external fluid medium, resulting in significant swelling and an initial increase in the volume of hybrid glass material (Fig. 3).

The biomechanical and biomimetic active scheme has two biomechanical functions, the first being strictly related to stabilization of the prosthetic system after implantation (the prosthesis can be loaded one hour after implantation), while the second function is associated with stimulation of bone growth exerted by implanting the bone surface from around her.

The volumetric expansion of the scaffolding can be effective in improving the stability of the primary implant, confirming the high bioactivity of the nanocomposite material tested (Fig. 4), (Petrescu and Petrescu, 2019; Petrescu, 2019).

The presence of the inflatable implant component, which is supported by the upper part of the Ti core of the implant, increases the removal torque after implantation when the system is in the presence of organic fluids.

The removal time, measured at different times after implantation, actually increased by more than $100 \%$ to 24 . In addition, even after one hour, the removal torque 
already increased from 43 to $62 \mathrm{~N}$ (an improvement of approximately $25 \%$ ). It has been described in a previous paper (Aversa et al., 2016b) that improved retention has immediately followed the spinning kinetics of the hybrid material scaffold (bottom of Fig. 4).

This increase in implant stability is due to the strong compression strains generated in the swollen rubber hybrid scaffold, as can be deduced from the map of the stained strains reported on the right side of Fig. 3 (the red color of the implant hybrid insert). The implant is then constrained in its socket by the external bone, which then increases the retention and stability of the implant. Applying a larger removal torque for explants is, therefore, necessary to increase the experimentally measured inflation rate.

Two biomechanical functions:

- Implant fixture

- Bone growth stimulus

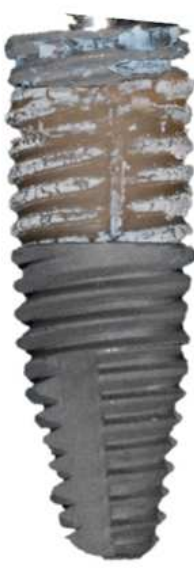

Dry glassy

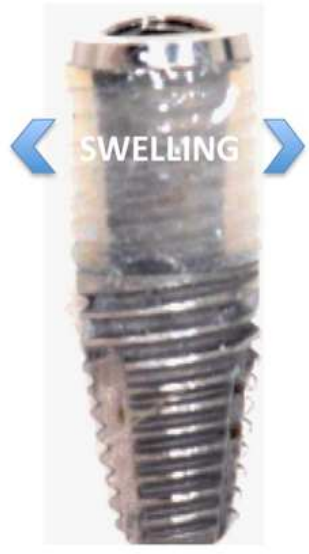

Swollen rubbery $13.5 \%$ linear

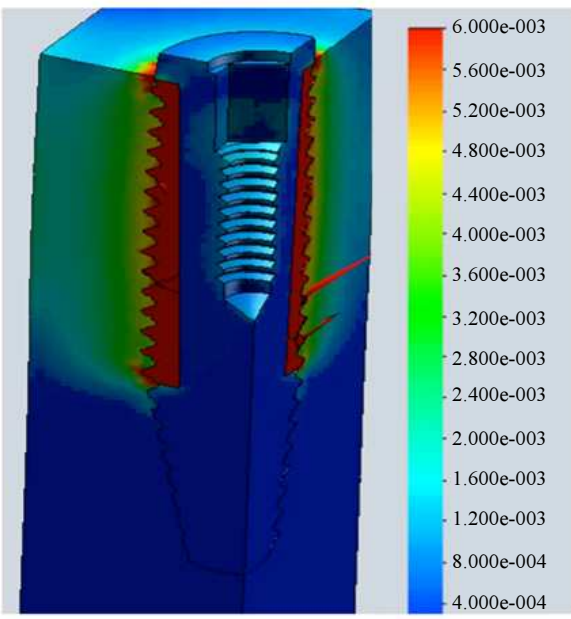

FEM simulation of swelling stresses

Fig. 3: Mechanisms of primary stability and osteoinduction improvements in Hybrid swellable scaffold modified Titanium implant. Glassy dry scaffold (left)

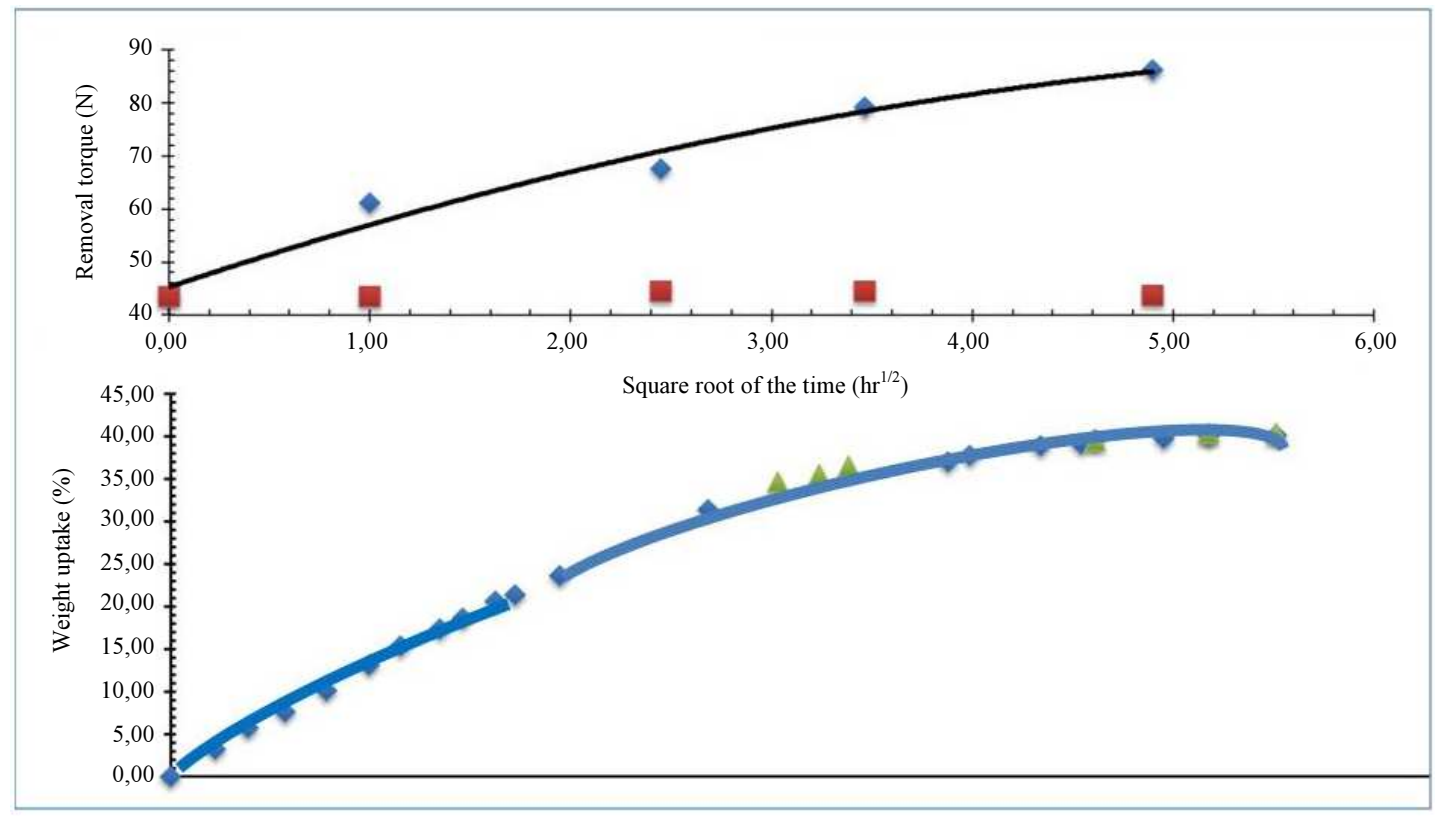

Fig. 4: Physiological fluid uptake (bottom) and implant stability improvement (top) of Hybrid swellable scaffold modified Titanium implant 
Furthermore, the constraint bone is subject to expansion strains as indicated by the colored map of the Von Mises strains reported in Fig. 3. The color scale used for this map is the same as that reported in Fig. 2, the physiology in which healthy growth and induction correspond to colors of yellow and green and blue and red to bone reabsorption.

The surrounding bones are subjected to healthy bone deformation at a distance equivalent to the diameter of the implant. In this toroidal volume surrounding the implant, an osteoinductive effect and a faster osteointegration of the implant are expected.

A micro-computer tomography confirmed these expectations.

Figure 5 shows the micro $\mathrm{CT}$ of these volumes.

The upper part of the figure reports bone reconstruction and external implants, while the lower part shows the 3D reconstruction of the volume surrounding an implant.

BIC and relative bone density had similar characteristics at cortical (a) and medullary (b) levels indicating good osteointegration of the initial bone implant. The newly formed bone near the implanted implants has similar characteristics to the preceding (c), indicating the biomechanical stimulation effect of the swollen and swollen hybrid material.

Traditional bone prostheses are mainly made of metals and ceramics with remarkable strength and stiffness, but with high physiological invasiveness.

These implants, which are expected to serve for a longer period of time, without failure or surgical revision, although they guarantee mechanical and functional wood, occur frequently physiologically and mechanically with the human bone. Failures and lack of long-term reliability result from incomplete integration of the personal characteristics of the bones and the patient with local physiology.

To reduce implant invasiveness, a biomimetic approach is suggested; the implant is expected to have an "equivalent stiffness" (the combination of the elastic modulus of the material and the prosthetic shape) that matches that of a bone area where it is implanted.

The bone modulus varies in size from 4 to $30 \mathrm{GPa}$, depending on the bone type and the direction of measurement (bone orthotropy). Current implant implants are isotropic with higher rigidities than bone (for example, between 160 and $210 \mathrm{GPa}$ for titanium and steel alloys) that greatly alter the physiological distribution of bone stress.

This behavior prevents the transfer of biomechanically modified stress to the adjacent bone, resulting in bone resorption around the implant and, consequently, weakening of the implant (this effect is called stress reduction).

This unwanted biomechanical invasiveness is due to the fact that the bones are functional and structural complex entities composed of less rigid, open and dense and rigid materials that are combined to provide rigidity both in orthotropic forces and in cortical bone forces. Equivalent to the three-dimensional properties of bone tissue should be truly necessary for implanted prostheses to achieve complete integration into the host bone tissue (Aversa et al., 2019).

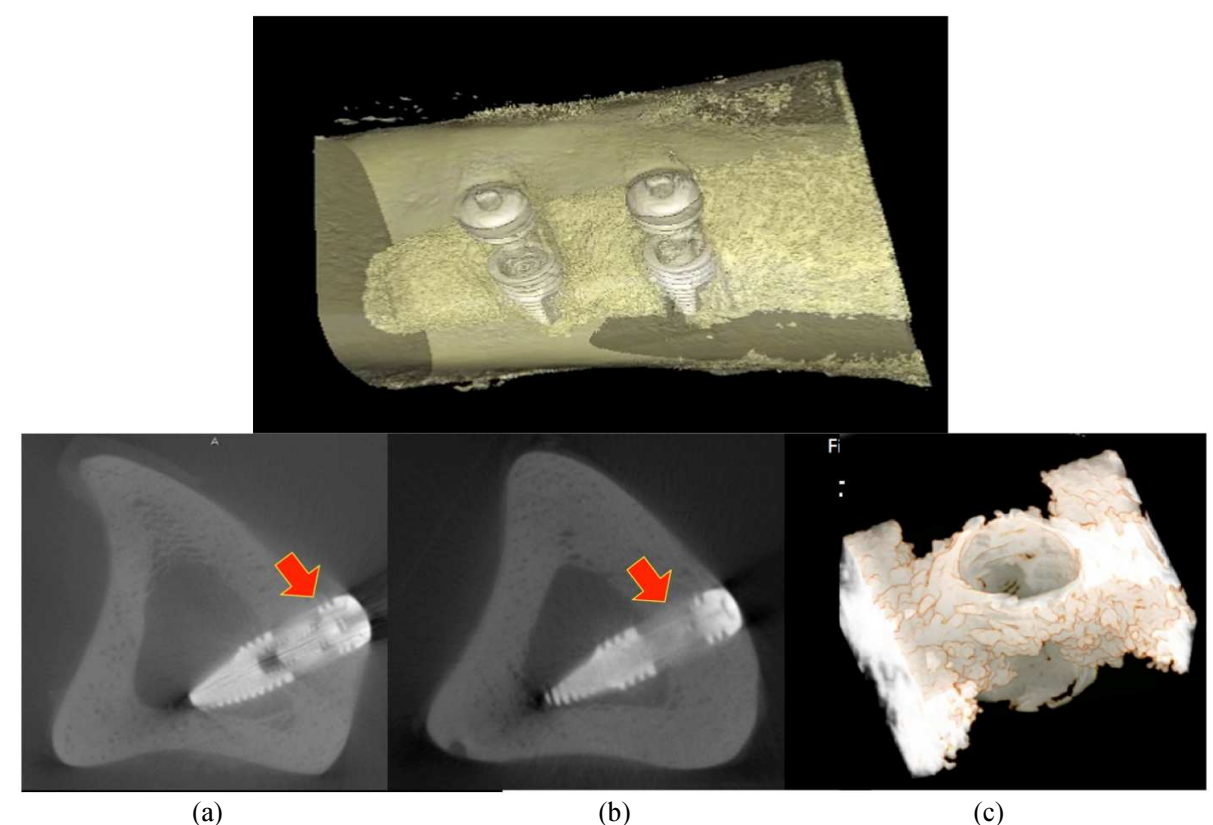

Fig. 5: The Bone to Implant Contact (BIC) and the relative bone density have shown similar characteristics at cortical (a) and medullar levels (b), Bone near to the implants shows similar characteristics (c) (Gramanzini et al., 2016) 


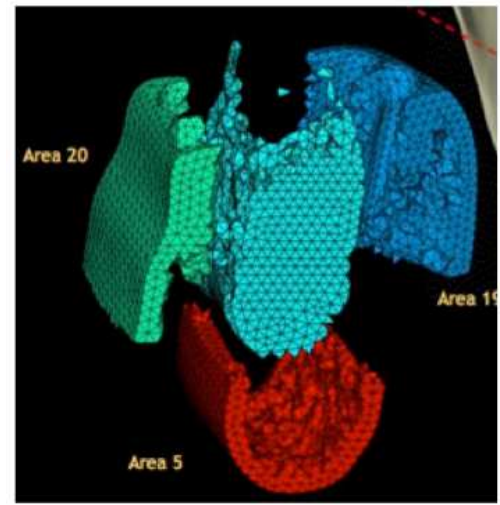

(a)

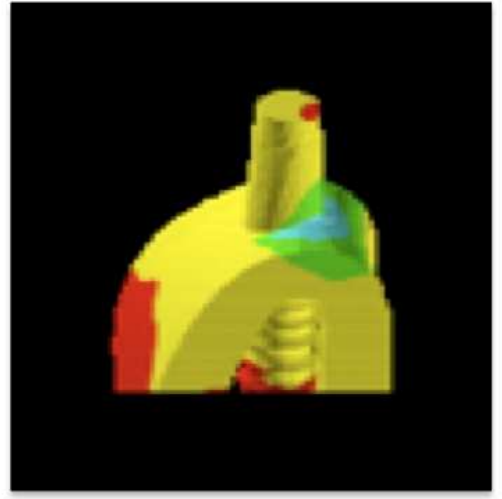

(b)

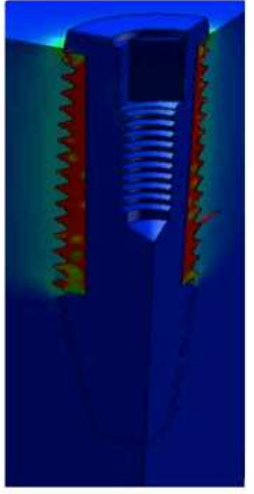

(c)

Fig. 6: Finite element analysis tools for biomechanical and biomimetic investigation: (a) Biofidel models of the bone, (b) Macro Finite element analysis of the implanted bone for definition of the stresses and strains physiological modifications, (c) Micro Finite Element Analysis at the bone-implant interface, (d) Strain limits for bone adaptive properties (Frost, 1994)

However, for the replacement and regeneration of soft tissues and osteoconductive and powerful mechanisms, porous and ceramic metallic biomaterials and polymeric systems and other metals have been proposed (Taylor et al., 2007; Parfitt, 1983; 1994; Martin et al., 1998).

From a biomechanical point of view, the structure must be rigid enough to support physiological tasks, but should not drastically exceed the stiffness of the replaced bone to avoid stress shielding. Attaching the implant to the surface or to the bone matrix should be improved by alternatives to reduce stress protection.

Clinical efficacy and long-term reliability of bone prostheses have been thoroughly investigated by analyzing finite elements to clarify the causes of the new replacement of invasive restoration (Gramanzini et al., 2016, Aversa et al., 2016a; 2016b).

Figure 6 summarizes the main methodological steps used in these studies: (a) biophysical bone for investigation, (b) highlighting and weighing fractured bone macroanalysis, (c) stress of microanalyses and strains on the bone-implant interface. The peak values and regions of adaptive bone properties are shown in Fig. 6d where unused, healthy and overloaded regimens are reported in Fig. 6d (Frost, 1990).

Bone prosthesis interference was recognized at two magnitude levels, namely a micro scale (bone-implant interface, Fig. 6c) and a macro scale (Fig. 6b).

The first small scale (Fig. 6c) explains the biological and micro-mechanical interactions of the synthetic biomaterial with bone-forming cells and highly adaptive adaptive properties, while the second on a macro scale (Fig. 6b) determines the complete biomechanical functionality of the implant material and the ability to restore the distribution of the biologic stress state in the prosthesis.

Osteoblasts under specific biochemical and mechanical stimuli mature and turn into osteocytes that mineralize the bone. The activity of osteoclasts under conditions that were not stimulated mechanically after prostheses could induce bone reabsorption in this new state of mechanical equilibrium.

Figure $6 \mathrm{~d}$ shows the adaptive bone properties that are in the coupling between bone formation and bone reabsorption.

This process refers to bone formation in which osteoclast reabsorption via osteoclasts and renewed osteoblast generations of precursors dynamically replace dynamics (Fig. 7).

The coupling can then be considered a complex mechanism of dynamic remodeling involving interactions of different types of cells and control stimuli. Mechanical stimulation should include physiological levels of the strain (Fig. 6d) between $50 \mathrm{~m}$ and $3000 \mathrm{~m}$ (Apicella et al., 2011; 2015; Aversa et al., 2009 ). Over 50 years of osteocyte activity predominate, resulting in bone resorption of between 1500 and 3000 $\mathrm{m}$, a slight increase in lamellar bone, predominantly over 3000 , with uncontrolled bone growth or resorption.

\section{Results and Discussion}

In the case of bone, which is a structural biological material that undergoes a mechanically induced continuous renewal (Fig. 7), the remodeling process is controlled by a dynamic balance involving osteoclasts (linking bone cells) and homeostatic renewal osteoblasts.

Osteoblasts under specific biochemical and mechanical stimuli may actually mature and turn into osteocytes that mineralize the bone. Instead, osteoclastic activity under conditions that were not stimulated mechanically after the prosthesis (i.e., in the stress release area) could induce bone reabsorption in this new state of bio-mechanical balance (Fig. 7).

Thus, in order to favor biomechanical integration and a longer maintenance period, a customized material, which carries a great combination of high strength and stiffness 
that fits the bone, must be used. The use of trabecular scaffolds and cortical bone to mimic behavior and colon have been proposed to recreate the distribution of macroscopic stress and bone deformities but requires a necessary micro-biomimetic interface that interacts with osteoblast osteoblasts. Nanomaterials Ceramic-polymer nanoparticles based on hydrophilic and ceramic polymers are potential candidates for new customizable biomaterials that will be used to cover porous phosphorus structures. The hybrid layer that comes into contact with bone can be customized by choosing nanophysics content and mechanical properties to achieve the biomechanical characteristics necessary to increase thickening of the osteoinductive bone in the porous structure of the metallic prosthesis. These resulting hybrid systems have the basic mechanical and biological properties that favor local healthy local generations. Specific and adjustable properties that simulate bone tissue that acts on the interface guide microparticle, leading to bone marrow growth and integration into host bone tissue. The main reasons for using the skeleton are therefore to provide a bone formation environment, maintain space and support the skeleton of the skeleton during the repair process.

New biomaterials that possess hybrid characteristics can be obtained by using nanometers in polymeric matrices (Aversa et al., 2016a; 2017a; 2017b), especially carbon nanofillers such as fullerenes and carbon nanotubes have been proposed to increase strength and stiffness nanocomposite materials. Although the use of carbon diamond nanofilm can further improve these properties, some technological challenges in production technology should be overcome.

Graphite is only one of allotropic carbon forms and is thermodynamically stable at ambient temperatures and pressures, while the diamond is another stable allotrope carbon at high pressures and at a temperature present in a metastable state at ambient and similar conditions (Petrescu and Calautit, 2016a; 2016b).

Differences in the stability of allotropic forms are a consequence of the large energy barrier separating the graphite Sp2 (left in Fig. 8) and diamond sp3 configuration (even in Fig. 8), which requires high temperatures and pressures in the presence of the transformation catalyst. However, an additional equilibrium parameter involving the surface becomes critical and significant for the distribution of the equilibrium energy to the nano dimension: Gibbs free energy is drastically influenced by the presence of surface energy input, modifying the phase diagram of the thermodynamic equilibrium (Badziag et al., 1990; Barnard et al., 2003; Barnard and Sternberg, 2007; Viecelli et al., 2001). Atomic models (Petrescu and Petrescu, 2019; Petrescu, 2019) have demonstrated that nanodiamonds with 3 nanometers with tetrahedral hydrocarbons are more stable than polyaromatic graphite under ambient conditions (Badziag et al., 1990; Aversa et al., 2016a; 2016b; 2016c; 2016d).

The presence of a more complex structure, generated at the nano-diamond interface, opens up new interesting technology applications. Cuboctodic structures of 1.03 to $3.0 \mathrm{~nm}$ with onion structure characterized by the passage from Sp3 to Sp2 carbon at their surfaces were observed by Barnard and Sternberg (2007).

A reversible reverse phase transformation in a nanodiamond-graphite cluster was observed by Xiao et al. (2014) appear in this morphological transition interface, which leads to daily bucky formation, characterized by a diamond core, a graphical case (schematized in Fig. 9) (Barnard and Sternberg, 2007). Such graphite nanodiamond surfaces can be modified using graphite carbon chemistry to form cyclohexane functional systems such as Diels-Alder cycle reactions between a conjugated diene and dienophiles (Jarre et al., 2011).

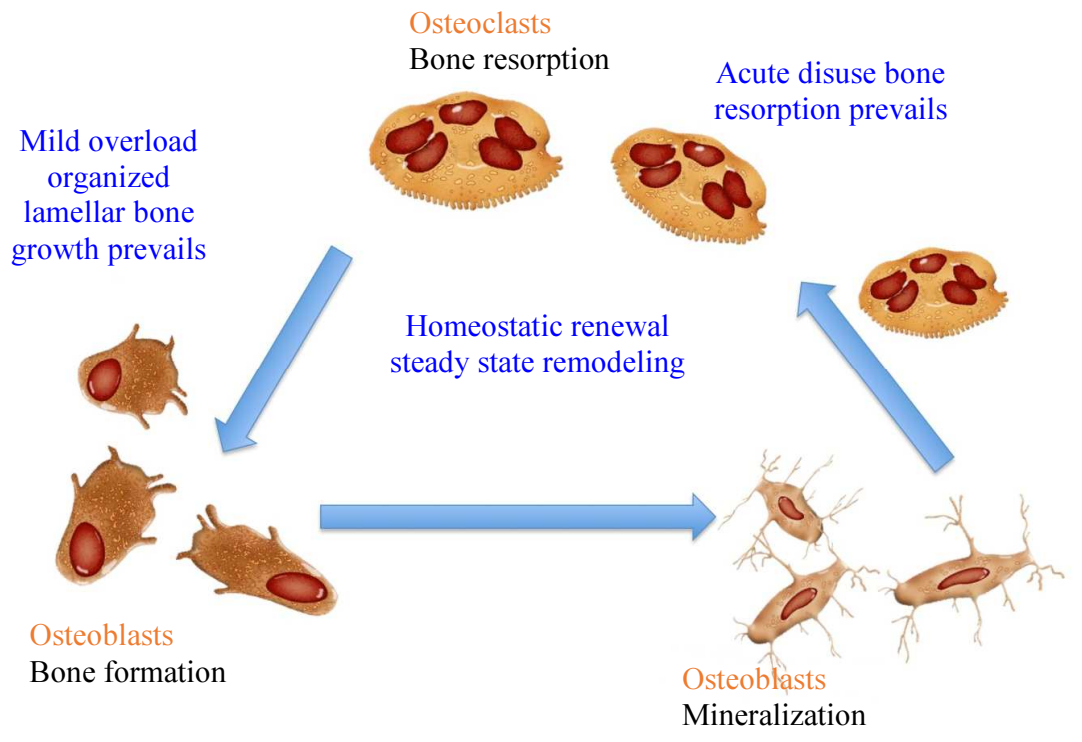

Fig. 7: Bone homeostasis mechanism involving Osteoblast, Osteocyte and Osteoclast cells 


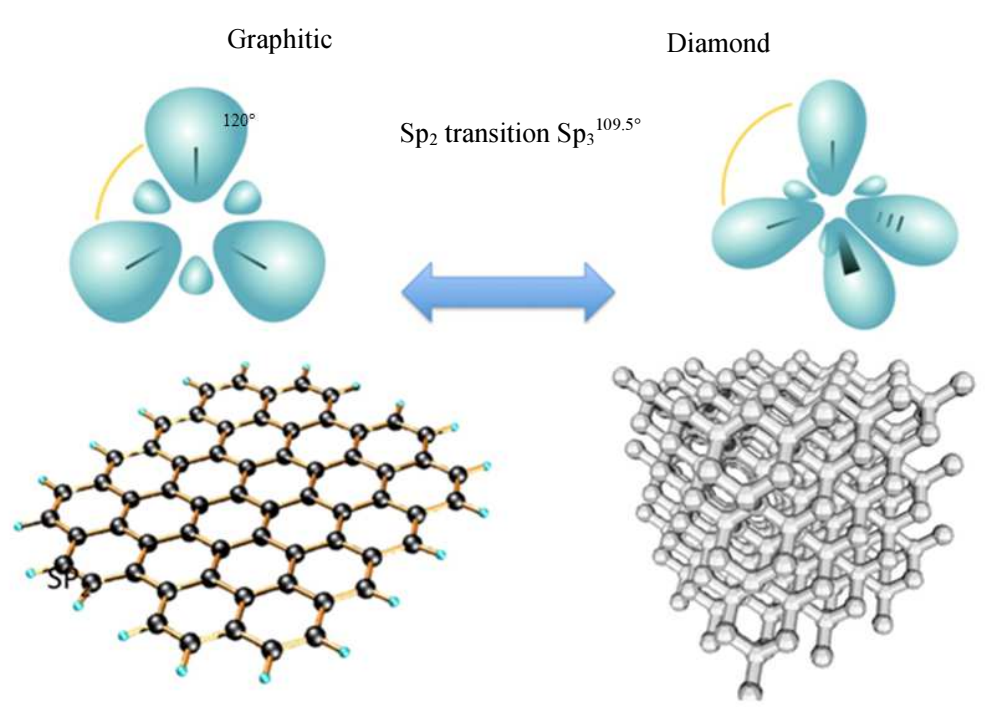

Fig. 8: (Left) Graphite (SP2 hybridization) and (Right) Diamond (SP3 hybridization) Carbon allotropic forms

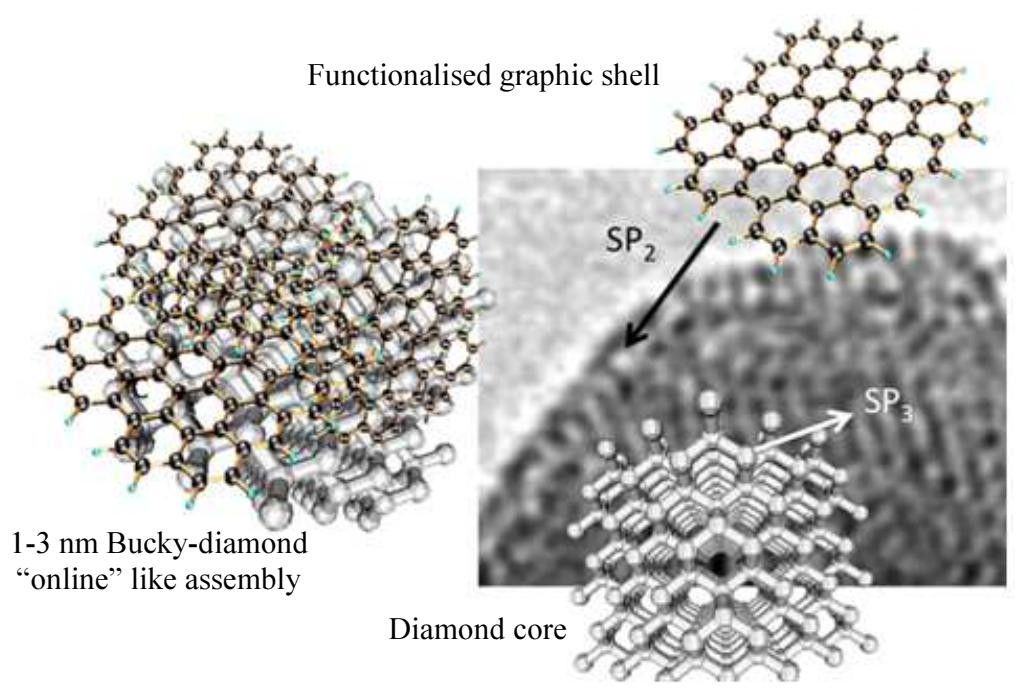

Fig. 9: Bucky-Diamond "onion" like cluster (left side): Nano-diamond core (lower right side) with external graphitic shell (upper right side) and TEM of a detonation nano-diamond atomic structure (right side)

The nano-crystalline $\mathrm{Sp} 2$ and $\mathrm{Sp} 3$ Carbon structures open up a new perspective for future technological development in structural biomedical applications. The nanocrystalline particles produced by the detonation of carbon explosive materials (Greiner et al., 1988; Ozawa et al., 2007; Chang et al., 2008) exhibit characteristic dimensions of 3-5 $\mathrm{nm}$.

Lubricants, galvanic coatings, polymer nanoparticles, polishing systems and niche applications recently used for electronics, emission devices, catalysts and combustion cells as nanocomposite membranes that lead protons for the application of detonating nanodiamonds have been proposed.

However, preliminary clinical and biochemical investigations have shown that these detonating nano- diamonds are biocompatible and non-toxic, opening up new biomedical applications, taking into account both the variety of surface chemical light and the intrinsic mechanical characteristics.

The detonation of nano-diamonds, characterized by different levels of purity and the presence of unwanted functional groups/elements, can not be directly appropriate for biomedical applications in which chemical purity and chemical uniformity of the surface (Lai and Barnard, 2011a; 2011b). After raw production, these materials are subjected to purification procedures. A simple method uses surface oxidation and different levels of purity and surface properties can be obtained. Oxidation carried out at elevated temperatures in an air/ozone atmosphere can lead to a purification of the 
carbon fraction not present as diamond up to $95 \%$ by weight (Osswald et al., 2006; Shenderova et al., 2011).

Oxidation of nano-diamond surfaces other than elimination of unwanted functional compounds forms oxygen-containing groups (the blue dots in Fig. 8 and 9) such as anhydrides and carboxylic acids (Shenderova et al., 2011) which are suitable for the polarization of hydrogen or polar with the appropriate species.

By purifying air/ozone, we can work on the very reactive and hydrophilic surface of the carboxylate, which is very suitable for biomedical applications (Krueger et al., 2008; 2006).

However, the toxicity of nano-diamonds remains controversial and is a real concern. In vitro and in vivo analyses are required to evaluate characteristics such as mechanical and physiological behaviors in vivo (Schrand et al., 2009a; 2009b; Zhang et al., 2011; Yuan et al., 2010). Although biocompatibility and negative effect have also been described in the literature on the use of nano silica particles, our published investigations have shown that nano-composite and hybrid materials made by combining amorphous silica nanoparticles have reached a high level of biocompatibility and micro-nanoparticles and p-HEMA.

These hydrogel hybrid nanocomposites have been tested for water sorption, water swelling and isotonic saline and for fibroblasts and osteoblasts as a cellular response with adhesion, distribution and morphology tests. The presence of polymer-bound silica makes these biomaterials excellent only for pHEMA. Good properties of osteoinduction were also observed for the differentiation of stem cells from the dental pulp Marrelli et al. (2015). These self-assembled nanostructured composites were also tested as a periimplanter scheme to match osteoblast growth factors or stem cells for osteoblast differentiation (Marrelli et al., 2015). This effect was mainly determined by micromechanical stress and the voltage generated at the bone/implant interface.

These new hybrid materials have been shown to be able to stimulate biomechanical bone growth within the range of physiological strains that allow for healthy growth to allow for complete early and organized integration of the implant into the receiving bone. Creating ideal scaffolds for bones is a growing argument for research. Such an ideal framework should provide a rigid and elastic mesh to temporarily replace damaged bone function while creating a bioactive substrate for bone regeneration, integrating it fully (Montheard et al., 1992; Kabra et al., 1991; Aversa et al., 2017a; 2017b).

\section{Preparation of Materials}

The $3 \mathrm{~nm}$ detonation nanoparticles (Aldrich, $\geq 97 \%$ ) with a specific surface area of $400 \mathrm{~m}^{2} \mathrm{~g}^{-1}$ were used as bioactive filling agents. Nanodiamond hydrophilization was performed (Shenderova et al., 2011; Aversa et al., 2016a; 2016b; 2016c; 2016d). The surface graft decomposition followed by functionality consists of the aggregate dispersion of $\sim 20 \mathrm{~nm}$. Treatment of hydrogen at high temperatures leading to nanoparticles Nanoparticles Nanoparticle nanoparticles of 2-4 nm was isolated by centrifugal isolation at $>10,000 \mathrm{rpm}$ (Shenderova et al., 2011).

The nanodispersion was mixed with 2-hydroxyethyl methacrylate monomers (HEMA) (Sigma-Aldrich Chemicals Co., St. Louis, MO, USA). The suspension of HEMA nanomaterials (Fig. 10) was radically polymerized in the presence of the azoisobutyronitrile thermal initiator (AIBN, Fluka Milano, Italy).

The nanocarbon content was prepared at 2 and $5 \%$ by volume.

The degassed reactive suspension was first transferred to $2.0 \mathrm{~mm}$ thick flat molds and then polymerized at $60^{\circ} \mathrm{C}$ for $24 \mathrm{~h}$. Finally, a cure at $90^{\circ} \mathrm{C}$ was obtained.

\section{Finite Element Analysis (FEA)}

Solid models of dental implants were generated using the Solidwork 2016 software. The titanium implant and the hybrid material replacing one part were molded. The FE model was obtained by importing solid models into ANSYS rel. 9.0 FEM software (Ansys Inc. Houston) using the IGES format. The volumes were linked to tetrahedral elements, resulting in a $3 \mathrm{D} F E$ model consisting of 31,240 elements and 35,841 nodes. The precision of the model was verified by the convergence test.

\section{Mechanical Characterization}

The effect of elasticity measurement on the dried, swollen and pendulous p-HEMA nanostructure was performed using a DTM METTLER-TOLEDO mechanical shear modulator (Zurich, Switzerland). The elastic and viscous components of the shear modulus were measured with a constant frequency in an isothermal state. The samples were vacuum dried at $60^{\circ} \mathrm{C}$ for $24 \mathrm{~h}$ before testing. In the shear test mode, discs of $10 \mathrm{~mm}$ diameter and $2 \mathrm{~mm}$ thick discs are placed between three steel plates forming a symmetrical sandwich. An isothermal scan was performed at $37^{\circ} \mathrm{C}$ in a nitrogen purged medium. Deformation control was set at $10 \mu \mathrm{m}$ and a force limit of $0.9 \mathrm{~N}$ was applied at an oscillating frequency of $10 \mathrm{~Hz}$ (Aversa et al., 2019).

The presence of the oxidized functional detonated nanodiamond in the monomer reaction mixture favors the self-orientation of HEMA polar monomers (Fig. 10). The nanoparticles are in fact characterized by the presence of oxygen-containing groups leading to a preferred orientation and self-assembly of the HEMA hydroxyl group monomers on the nanoparticle sizing surface (left side of Fig. 10). 
Nanosilica hybrid Nanocomposites have been described in a previous paper (Aversa et al., 2016a) to show the self-assembled analog behavior that led to the formation of nanostructured hybrid materials. Similarly, functional nanopowder that does not contain oxygen binding atoms (Fig. 10) and functional HEMA functional groups can produce monomer.

The polymerization of these silicates from HEMA/silica gel leads to the formation of a nanostructured hybrid material exhibiting specific and specific properties, such as improved mechanical stiffness and biocompatibility (Aversa et al., 2016a; Petrescu et al., 2016a; 2016b; 2016c).

Applying this model of nanostructure formation to the nanodiamond/HEMA suspension polymerization, a similar improvement in mechanical properties and biocompatibility is expected. However, improved mechanical properties are expected to be more relevant due to the high and high shear force (Azo technology).

The stiffness of the synthetic diamond may be up to 15 times higher than that of silicon, i.e. from the shear modulus of about $450 \mathrm{GPa}$ Vs about $30 \mathrm{GPa}$ (Azo). By acting as a filler or hybrid formation, nano-diamond detonation could generate the mechanical behavior of similar nanoparticle hybrids (Aversa et al., 2016a), the behavior of pure variation according to the volume fraction of hybrid diamond nanoparticles in the material could be assessed.

As described by Aversa et al. (2016a) aims at balancing PHEMA hybrid nano-silicas in physiological solutions. Water molecules that bind to polymeric hydrophilic groups induce a significant plasticization of the nanocomposite (in our case, a $16 \%$ by volume nanocomposite) that reduces the shear modulus of the dried initial samples from 8-9 GPa to 0.01-1, $1 \mathrm{GPa} 6$, Aversa et al. (2016d; 2019).

The compositional dependence of PHEMA impulse shear modulus is not described by the classical HalpinTsai equation (Halpin and Kardos, 1976) valid for particle composites, but a linear dependence is observed. These findings confirmed the hybrid character of pHEMA nano-silica compounds.

On the other hand, the same stiffness can be achieved at a much lower volumetric loading by using the nanodiamond, i.e., between 1 and $5 \%$ in the dry state.

Figure 10 shows a schematic of self-assembled PHEMA nanodiamonds, while Fig. 11 compares the shear modulus that can be obtained using nanoparticulate amorphous particles and nano-diamond crystalline particles. The red points are the 2 and 5\% nano-diamond shear modulus, measured in the shear mode using a dynamic mechanical tester (Aversa et al., 2017b).

These preliminary tests have confirmed our estimated theoretical values for hybrid configurations.

Therefore, the adaptive properties of the bones can benefit from the use of biomechanical and biomechanical materials (biomimetics).

\section{"In vivo" Evaluation of Bioactivity and Osteoinduction of Implants}

The in vivo study aimed at assessing the bioactivity and osteoinductivity of the ceramic-polymer hybrid scaffold was presented by Aversa et al. (2016d). Unmodified titanium dental implants and siliconized nano-hybrid implants were tested for 2 months at the laboratory hamster femur. Micro-tomography was performed to evaluate bone density and distribution around the implant (Aversa et al., 2019).

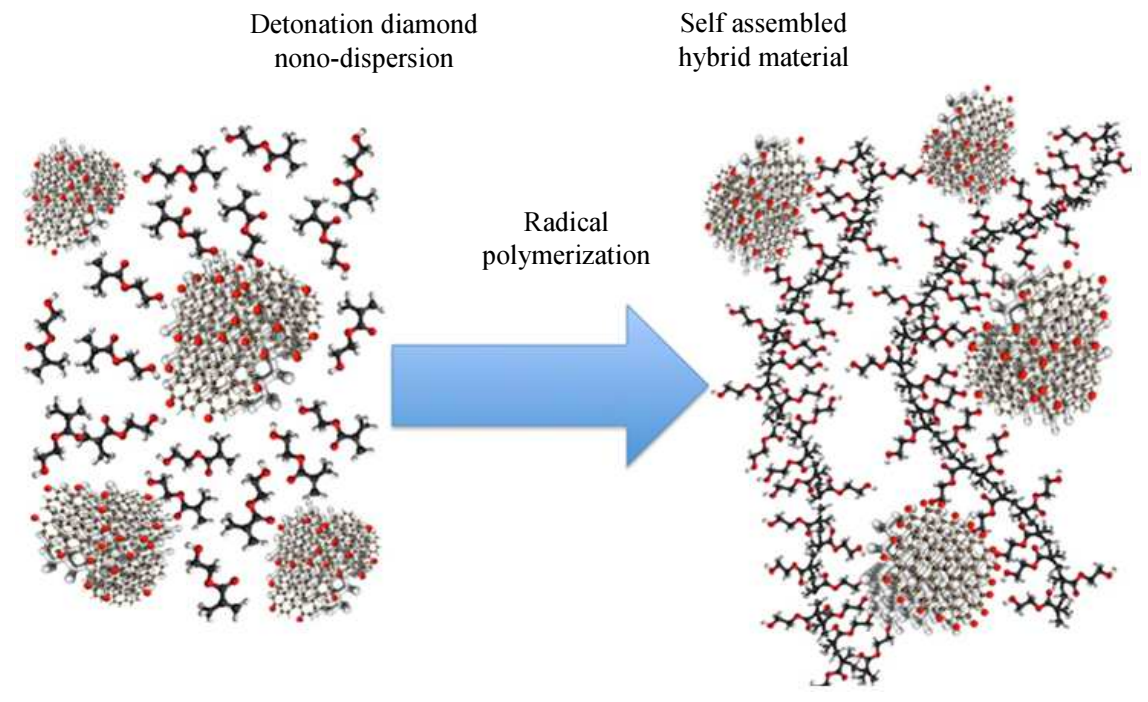

Fig. 10: Self-assembly of HEMA monomers in presence of functionalised Detonation Nano-diamond (left side) and hypothesized Nanodiamonds pHEMA self assembled hybrid nanocomposite (right side) 


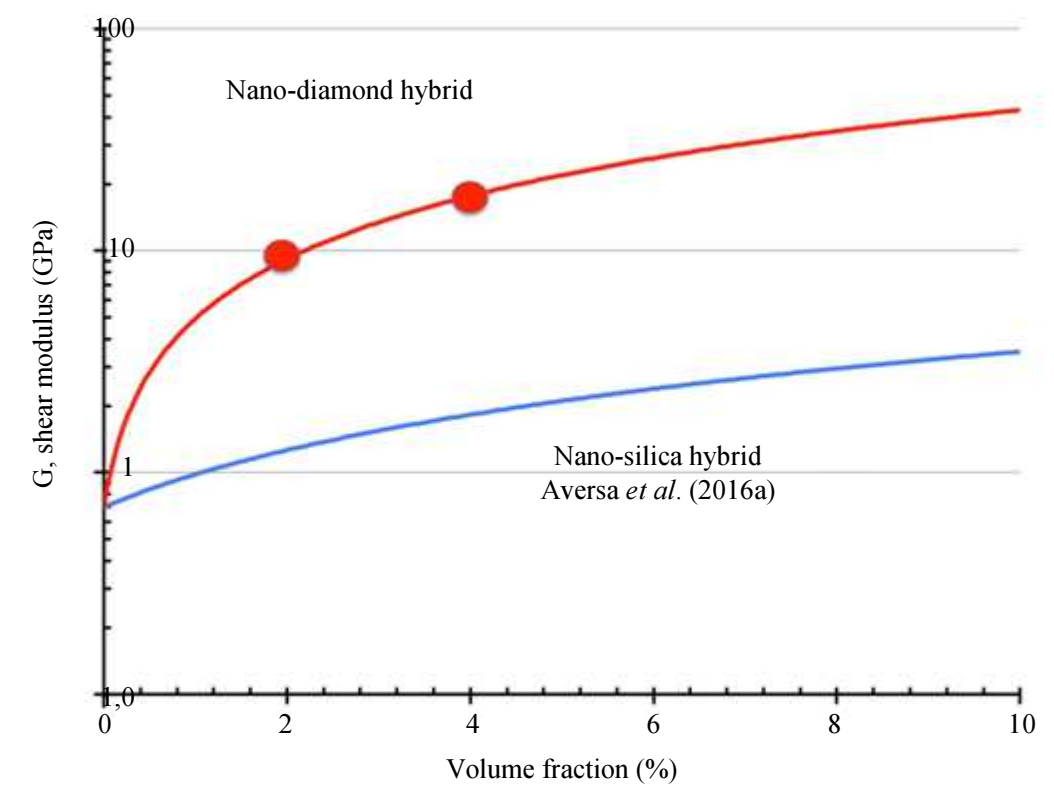

Fig. 11: Camparison of mechanical shear properties on amorphous nanosilica and crystalline nano-diamonds-pHEMA hybrid nanocomposite
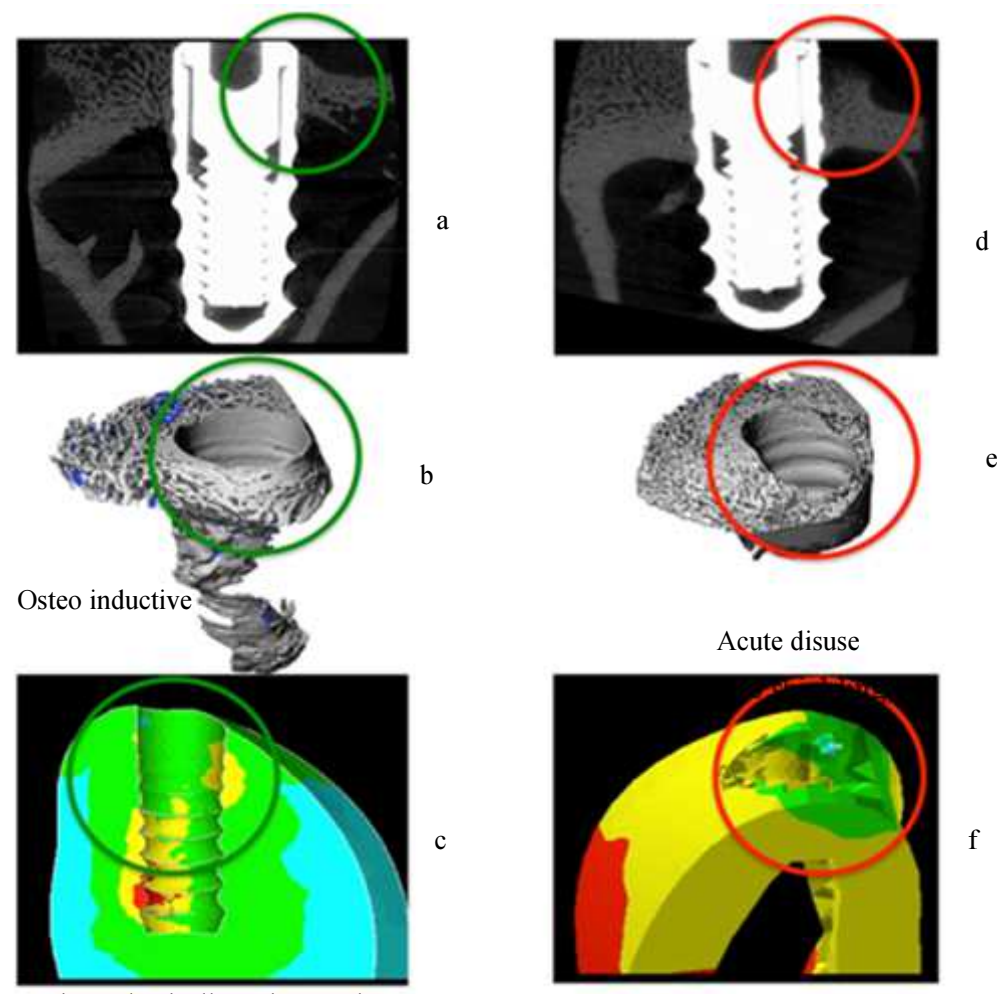

Acute disuse

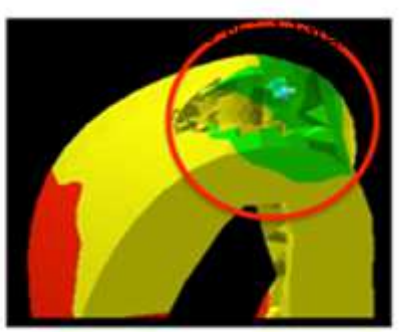

Uncoated

Fig. 12: Comparison of "in vivo" and Finite Element Biomechanical Analysis results on a dental implant with and without the biomechanically and osteoinductive hybrid coating

The biomechanical and active osteoinductive characteristic of hybrid materials is summarized in Fig. 12, in which microscopic microscopy and bone reconstruction and biomechanical analysis by finite element analysis are compared for the same active (left) and non-right biomechanical implants. 
Active biomechanical coverage (100 microns) demonstrated that it is able to transfer the physiological strains necessary to avoid stress protection and bone resorption in the vicinity of the implant neck (the green cross section of Fig. 12a-12c).

The explant implant (on the right side of Fig. 12) shows a significant bone resorption process in the same area (red circulating in Fig. 12d-12f), which was correctly predicted using the biofilm model (Fig. 6b) for the final bone implant (Fig. 12f).

FEM analysis in the same area of the hybrid biomaterial coated implant predicted a more physiological stress distribution due to the active biomechanical interface that stimulates osteoblast growth (Fig. 12c).

Commercial 2-hydroxyethyl methacrylate was purchased from Sigma-Aldrich Chemicals Co., (St. Louis, MO, USA). Smoke silicon dioxide (Aerosil 300 Degussa, Germany) with an average diameter of $7 \mathrm{~nm}$ and a specific surface area of $300 \mathrm{~m}^{2} \mathrm{~g}^{-1}$ was used as a bioactive filler. The initiator, $\alpha-\alpha$ 'azoisobutyronitrile (AIBN), was purchased from Fluka (Milan, Italy). HEMA monomers with an increasing amount of chemical silica (4 to $30 \%$ by volume) were mixed. The resin was poured into $2 \mathrm{~mm}$ flat plates, polymerized in an air circulation oven set at $60^{\circ} \mathrm{C}$ for $24 \mathrm{~h}$ and finally treated at $90^{\circ} \mathrm{C}$ for $1 \mathrm{~h}$.

Planar samples were used for sorption and swelling experiments with aqueous isotonic saline $(0.15 \mathrm{M} \mathrm{NaCl})$. The aqueous solution absorbed in the initially dried samples was determined at equilibrium by gravimetric measurements in a $0.1 \mathrm{mg}$ Mettler Toledo balance sheet (Milano, Italy). Advanced inflation left in the abnormal sorption of sample II was monitored by measuring the time of thickness of the non-deposited residual glass core. The sorption and balance swelling experiments were performed at $37^{\circ} \mathrm{C}$ (thermostatic water bath) until the monitoring of the constant weight absorption monitoring $(100 \mathrm{~h})$ was monitored.

Solid dental implant models were created using the Solidwork 2007 software. Titanium implant and hybrid material replacing one part were molded. The FE model was obtained by importing solid models into ANSYS rel. 9.0 FEM software (Ansys Inc. Houston) using the IGES format. The volumes were linked to tetrahedral elements, resulting in a 3D FE model consisting of 31,240 of 309 elements and 35,841 nodes. The precision of the model was verified by convergence tests.

Measurement of the elastic modulus by sampling the hybrid nanocomposite and the swollen p-HEMA hybrid was performed using a mechanical shear METTLERTOLEDO (DMA) (Zurich, Switzerland). The elastic and viscous components of the shear modulus were measured with a constant frequency in an isothermal state. The samples were vacuum dried at $60^{\circ} \mathrm{C}$ for $24 \mathrm{~h}$ before testing. In shear mode, discs of $10 \mathrm{~mm}$ diameter and $2 \mathrm{~mm}$ thick discs are placed between three steel plates forming a symmetrical sandwich. An isothermal scan at $37^{\circ} \mathrm{C}$ was carried out in a nitrogen purged medium. The deformation control was set at $10 \mu \mathrm{m}$ and a force limit of $0.9 \mathrm{~N}$ was applied at an oscillating frequency of $10 \mathrm{~Hz}$.

The in vitro study aims to evaluate the potential of nanomaterials to improve the nanomaterials of nanomaterials Nanomaterials Nanomaterials (Aversa et al., 2016b; Sorrentino et al., 2007; 2009). Both the test body and the control body were randomly assigned to four groups; each test group consisted of 9 implants, while each control group consisted of three fixed elements. Insertion values ranging between 43.4 and 44.5 and between 44.2 and $45.7 \mathrm{Ncm}$ were recorded in the control and control group. In groups 1 through 4 and in groups 5 to 8 , the implants were removed after $1,6,12$ and $24 \mathrm{~h}$. The values of the removal torque were recorded as described above. The in vivo study aims at assessing the bioactivity and osteoinductivity of the ceramic-polymer hybrid scaffold. Dental prostheses Unmodified titanium and modified and coated prostheses were implanted into the rabbit femur and eliminated after two months. The micro-calculus tomography was performed on the explanted femur and the bone density and distribution on the implant was evaluated.

Our research aims at designing a completed biomimetic dental implant to stimulate normal OB growth in adaptable mandibular bones. To achieve this result, both a suitable biomimetic scheme material and an external implant screw piece should be designed.

The biomimetic feature of our hybrid materials has been investigated for both mechanical properties and swelling properties. The physiological behavior of the bone material to be imitated from the bioactive material of the scaffold refers to the following aspects:

- Mechanical properties (dry and swollen)

- Bioactivity (in vivo implant)

The PhEMA nanocomposite hybrid components with a concentration of 4 to $30 \%$ silicon dioxide volume were mechanically mechanically mechanically tested isometric, operating in a nitrogen atmosphere at $10 \mathrm{~Hz}$ and $37^{\circ} \mathrm{C}$ predominantly viscous viscosity of all compositions. The measured shear modulus is shown in Fig. 13. The measured shear modulus of PHEMANanosilica composites does not follow the classical Halpin-Tsai equation for particulate composites (the ascending line shown in Fig. 13).

A linear dependence of the shear modulus values on the progressive loading of nano silicon was observed. This unexpected behavior indicates the hybrid nature of PHEMA nano-silica composites.

To determine the appropriate nanofiller/p-HEMA ratio of the appropriate potential hybrid nanocomposite, the requirements of the target properties are: Similar to bone rigidity during implantation. 


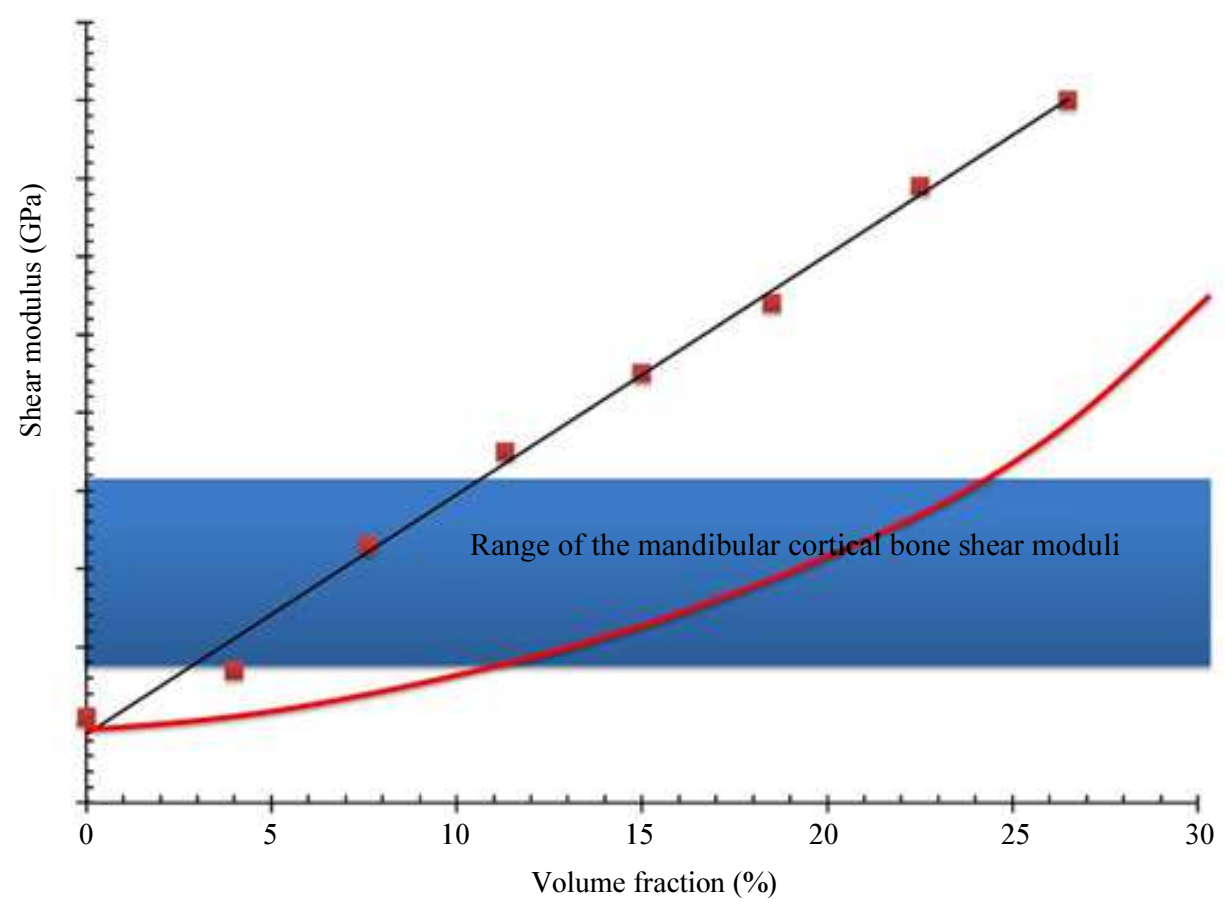

Fig. 13: Shear moduli of the hybrid nanocomposites at different nano silica filler loading. The theoretical Halpin-Tsai curve is reported for comparison in the figure

Shear modulators comparable to those of cortical bone were measured dry for nano silicon fractions ranging from 4 to $12 \%$. A $5 \%$ volume fraction was then chosen for sample preparation and FEA simulations (Aversa et al., 2016b; 2016c; 2016d).

Elastic modulating (traction test) ranging from 2-20 MPa (strain-curing effect) was measured for the swollen hybrid composite ( $5 \%$ by volume). This value becomes comparable to that of the tense periodontal ligament under the same conditions as articular cartilage.

The 5\% hybrid nano-composite swells dramatically in isotonic aqueous solution, raising $50 \%$ of its dry weight while reducing the shear modulus to 2-3 MPa (measured in DMA at $10 \mathrm{~Hz}$ ). Such a phenomenon is associated with plasticization of the water-induced polymer that reduces the transition temperature of the polymer glass below the test temperature 311 . The sorption behavior was investigated in a $0.15 \mathrm{M}$ isotonic isotonic isotonic solution maintained at $37^{\circ} \mathrm{C}$ by hybrids of glass with a volume fraction of $5 \%$ both for the weight absorption of the solution and for the swollen kinetics. After exposure to the aqueous solution, $2 \mathrm{~mm}$ thick coated PHEMA glass plates begin to swell with a clear face dividing the outer rubber portion and the untreated glass core. The thickness of the correct glass progressively reduces the front feed through the sample. A measure of the swollen kinetics, which was reported in Fig. 14 based on the square root of time, is given by the rate of reduction of the glass core as a function of time.
The swollen face has initially advanced at a constant rate, according to the relaxed relaxed relaxed mechanism of anomalies, indicated as "case II sorption".

An initial linear inflation rate is approximately 0.10 $\mathrm{mm}$ per hour. Since the swelling continues, however, the diffuse resistance develops into the exfoliated outer skin, resulting in diffusion-controlled swelling of the remaining glass core. When inflated fronts meet, the weight gain of the samples is about $27 \%$ but continues to increase to a balance of $40 \%$. This is due to complete balancing through the thickness of the sample. At steady state, a $14.5 \%$ increase in sample thickness and a $50 \%$ increase in volume were measured. These values were used to evaluate by simulating the analysis of finite elements the dimensional changes that appear in the complex geometry of the modified dental implant described in the following paragraph.

The use of the active and biocompatible biomechanical interface has been "engineered" to reproduce bone distribution compatible with the biomimetic. Intervals of physiological strains and adaptive bone properties are reported. There are superior surfaces $(>3000 \mathrm{~m})(<50 \mathrm{~m})$ that do not favor healthy bone growth. The variable tension of adaptive bone growth is bone resorption $(<50$ $\mathrm{m})$, remodeling $(50-1500 \mathrm{~m})$, organized growth $(1500-$ $3000 \mathrm{~m}$ ), resorption (> $3000 \mathrm{~m})$.

Biomimetic aspects are investigated using hybrid osteoconductive nano-composite coupled with FEM modeling of swelling and deformation of the hybrid 
material. The proposed solid CAD model of the new ceramic-polymer modified implant is shown in Fig. 15.

Two biomechanical functions were considered during implant design: Fixation of the implant and stimulation of bone growth. A portion of the Ti screw was replaced by the hybrid nano-composite that maintains the continuity of the outer yarns, while the interior, in which the remaining Ti core is conical through the threaded tip, has a thickness of between 0.5 and $0.8 \mathrm{~mm}$.

This thickening of the hybrid ceramic-polymer hybrid nanocomposite produces, following inflation, a progressive volumetric increase of the peak. The scaffold should play two biomechanical functions: One structural as part of the fixation device and one bioactive as a stimulation stimulus of the bone. From a physiological point of view, stretching the periodontal ligament causes the new bone to fall into the toothbrush. Since the modulus of elasticity of the swollen scaffold was comparable to that of the periodontal ligament, the analysis of the finite element confirmed that swelling of the nanocomposite could act as a biomechanical entry of bone growth.

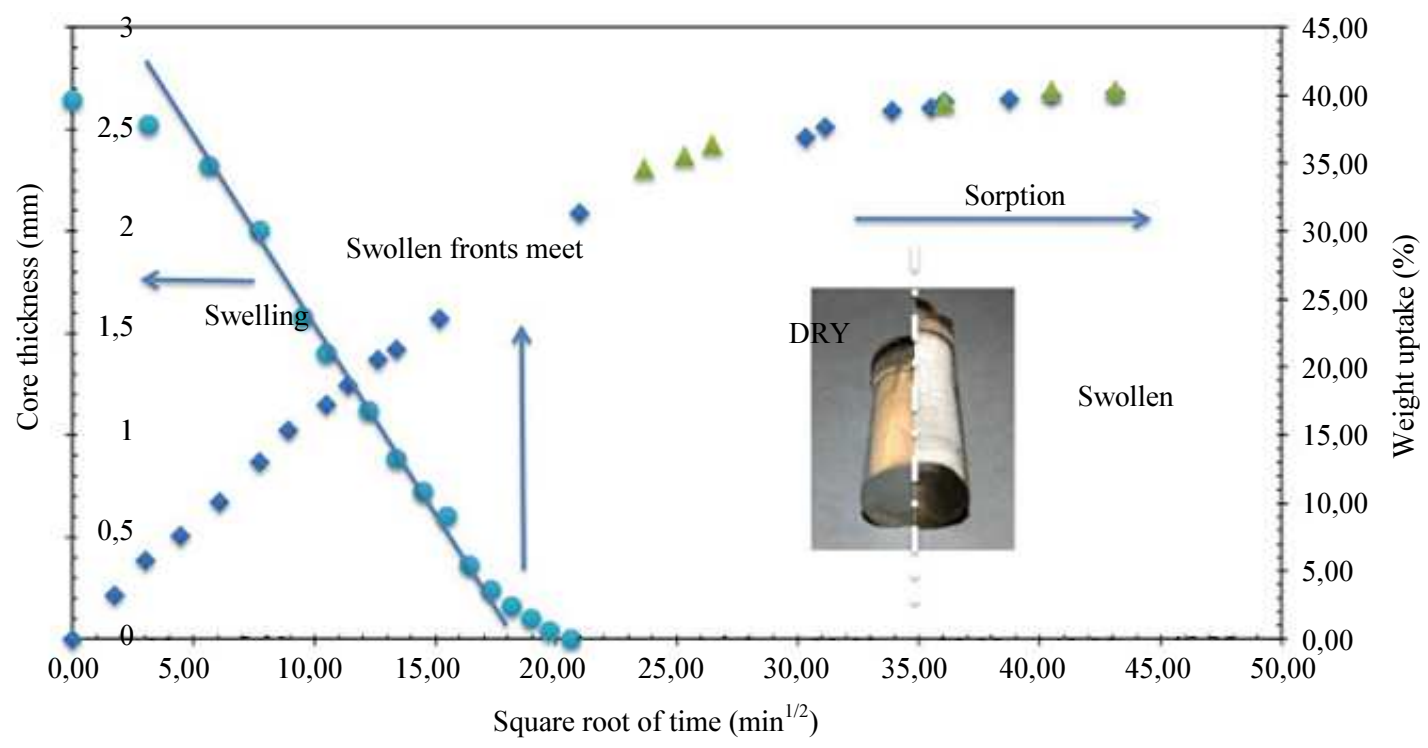

Fig. 14: Swelling and sorption kinetics of a $5 \%$ by volume hybrid nanocomposite in $0.15 \mathrm{M} \mathrm{NaCl}$ water solution (isotonic)

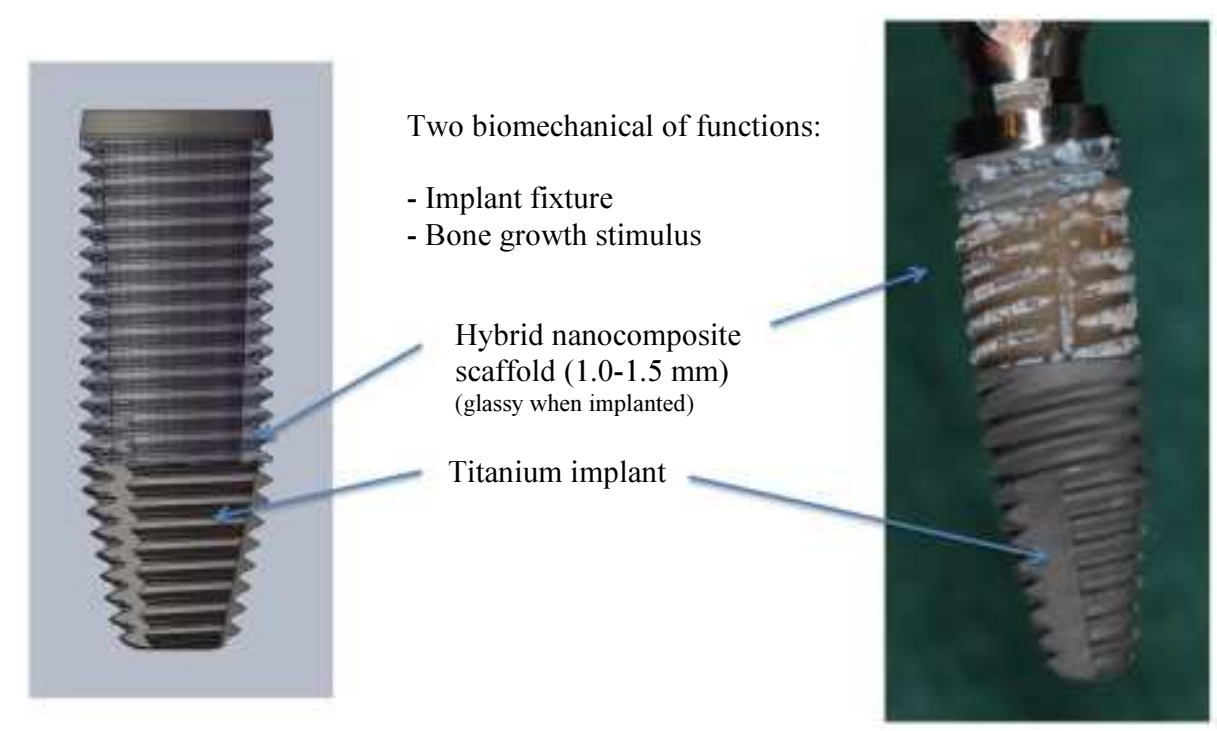

Fig. 15: Thick elastic scaffold hybrid material mimicking periodontal ligament functions in the Biomimetic implant: CAD solid model and a prototype for use in "in vivo" tests of the new ceramic-polymeric modified Titanium implant 


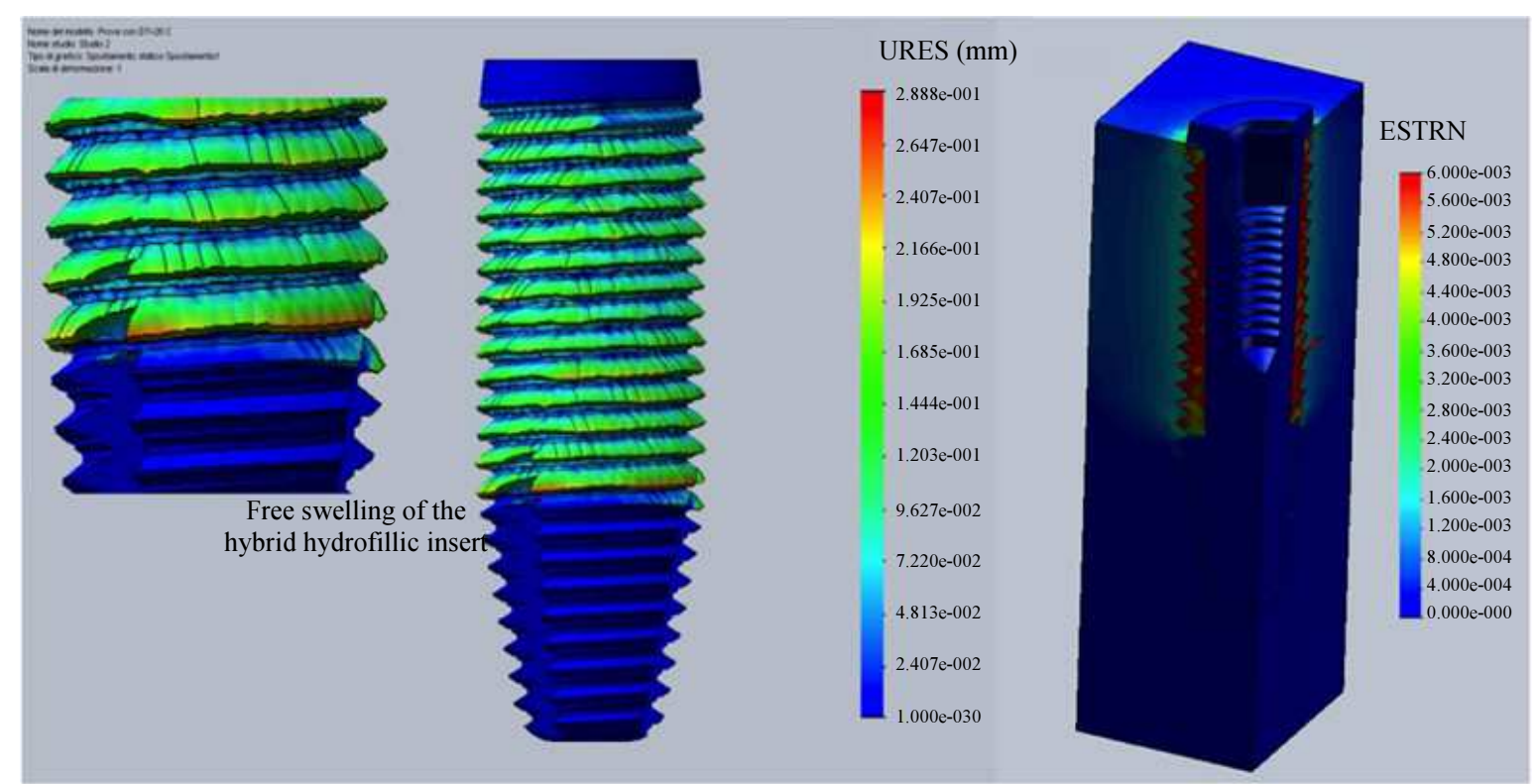

Fig. 16: Displacements (URES) in mm and strains (ESTRN) of the hybrid ceramic-polymeric insert undergoing physiological fluid free and constrained swelling after implantation

Figure 16 shows the results of a finite element analysis performed on the new implant simulating the swelling of the polymer-ceramic polymer insert in a physiological fluid: Displacements up to $0.2 \mathrm{~mm}$ were calculated (after 8 hours depending on the swelling velocity of $0.1 \mathrm{~mm} / \mathrm{h}$ ). This occurrence favors fixation and stabilization of the prosthetic device after implantation (the first biomechanical function).

Moreover, since remodeling is not triggered by the main stress but by dynamic loads (not static charges) on remodeling, a positive growth stimulus is due to the presence of a deformed hybrid at a physiological compression of 5-30 m; and stress stress during bone healing and integration of the bone implant (second biomechanical function), (Aversa et al., 2016a; 2019).

The blooming of the ceramic-polymer hybrid insert then sets the implant into the bone and creates an active biomechanical interface for bone augmentation. Bone stress was modulated by choosing a skeletal swollen thickness for healthy bone growth. The in vivo tests performed using this new modified oral implant confirmed the improved ability of these implants to promote early osteointegration.

This in vitro study aimed at assessing the possibility of improving the primary stability of oral implants through three-dimensional schemes consisting of a hybrid polymeric material of nanocomposite ceramic materials.

In the test groups, mean withdrawal rates increased progressively over time (diamond points) ranging from 61.2 after $1 \mathrm{~h}$ to $86.2 \mathrm{Ncm}$ after $24 \mathrm{~h}$, showing how swelling of the scaffold improved the stability of the primary implant. In contrast, in control groups (squares), the average values of the removal torque were between 43.7 and $44.9 \mathrm{Ncm}$.

Figure 17 compares the average growth of the removal torque and the sorption/inflation kinetics. The results of the Micro CT scan are shown in Fig. 18. Thin hybrid implants showed two main differences between osteoinductivity and bioactivity compared to unmodified implants:

- Avoiding bone resorption in the cortical bone surrounding the implant neck

- Improving osteoinduction in the bone marrow

Immediate titanium implants showed significant bone resorption in the cortical bone.

This effect may be related to the biomechanical stimulation of incorrect cortical bones outside the range of 50-3000 m. To avoid this undesirable effect due to a proper mechanical stimulus induced by the interface of the hybrid bioactive hybrid material.

Furthermore, bone growth on the implant surface (the left implant in Fig. 18) is due to the osteoinductivity of the hybrid skeleton material tested (Aversa et al., 2016b; 2016c; 2016d).

Biomaterials Today's science is a very interdisciplinary field that plays a central role in the development of tissue engineering applications involving close collaboration between biologists, chemists, material engineers, physicists and clinicians who have researched in this area at a favorable level to new development systems. Regenerative medicine has developed a lot and will guarantee many pat-to-bed applications (Montheard et al., 1992; Filmon et al., 2002; 
Davis et al., 1991; Kabra et al., 1991; Apicella et al., Peluso et al., 1997; Chow et al., 2010).

Biomimetic Ability (Apicella and Hopfenberg, 1982; Apicella et al., 2010; 2011; 2015; Aversa et al., 2009; Perillo et al., 2010; Petrescu et al., 2015; SchwartzDabney and Dechow, 2003; Töyräsa et al., 2001; Frost, 1990; Gramanzini et al., 2016; Holley et al., 1970; Nicolais et al., 1984; Aversa et al., 2016a).
The new hybrid nanoparticles are prepared by polymerizing the hydroxy-ethyl-methacrylate monomers filled with detonating nanotubes (up to $5 \%$ by volume). This material absorbs water and swells in aqueous physiological solutions (up to $40-45 \%$ by weight), transforming it into glassy and rubbery conditions. Low levels of non-diamond loads can improve the mechanical properties of hybrid materials.
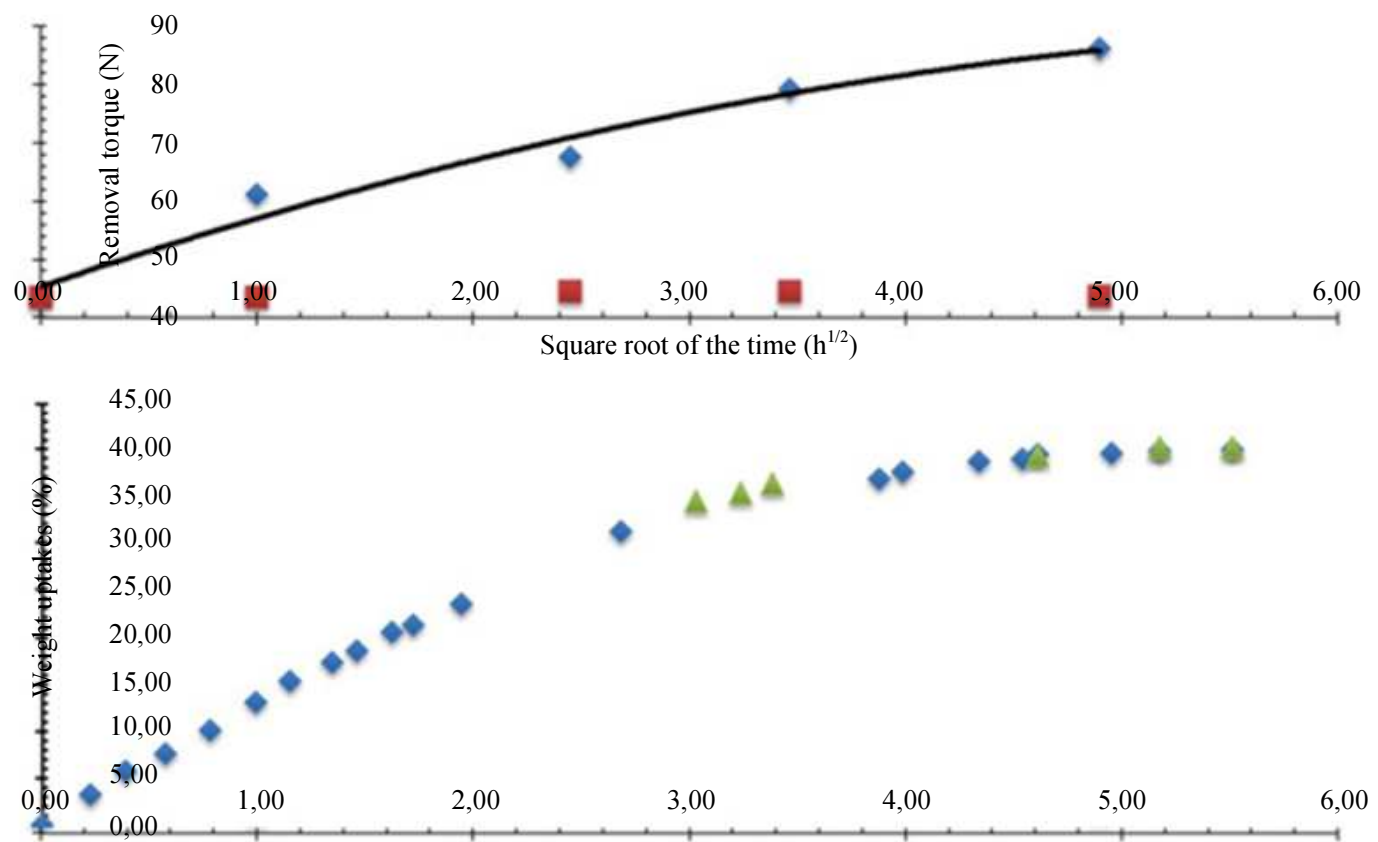

Fig. 17: Comparison between the kinetics of after implantation removal torque increases and physiological fluid uptakes in modified implants undergoing swelling

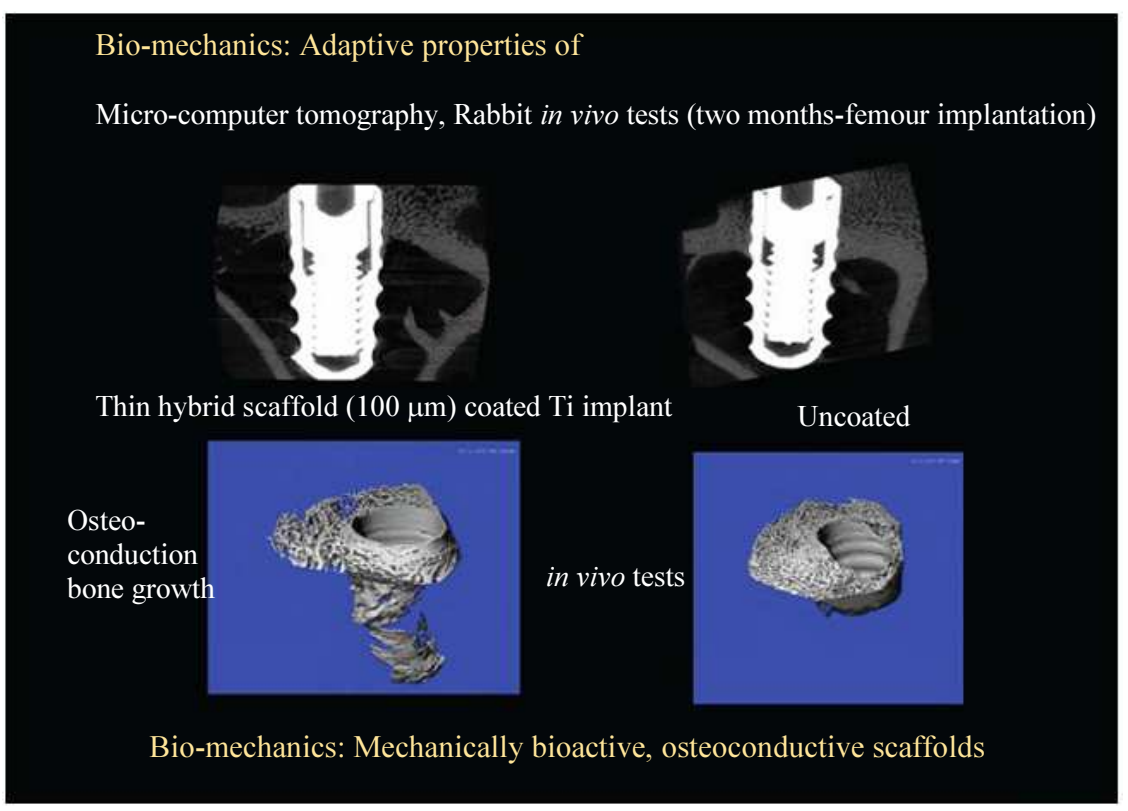

Fig. 18: Biomechanicanics in adaptive morphology of the bone and osteoinduction in the hybrid scaffolding materials 
Hydromechanically compatible hybrid hydrogels can be used as scaffolding materials to increase stress adaptation mechanisms to micro and macro prostheses.

The introduction of active biomechanical interfaces will improve biomimetic implantation while reproducing the biomechanical functions of cartilage and ligaments (Apicella and Hopfenberg, 1982; Apicella et al., 2010; 2011; 2015; Aversa et al., 2009; Schwartz-Dabney and Dechow, 2003, Töyräsa et al., 2001; Frost, 1990; Gramanzini et al., 2016; Holley et al., 1970; Nicolais et al., 1984).

The use of metallic microtrabecular prostheses (Aversa et al., 2016c; 2016d) coated with ceramicpolymeric hybrid scaffolds (Aversa et al., 2016d) was proposed to recreate macro and micro-distribution of stresses and deformations in the bone.

The development of polymer hybrid nanocomposites has been proposed in recent studies (Aversa et al., 2016b; 2016c; 2019). These hybrid materials can induce the mechanical and biological properties needed to promote healthy local generations.

The innovative features of a biomimetic approach are that the prosthesis is now designed to replace the joint, damaged by various causes, but does not stimulate tissue regeneration. Also, the average length of a prosthesis is about 10/15 years, while the new "biomimetic prosthesis" will last longer, estimated at 20/25 years.

This is very important because the average life span has increased significantly, increasing the number of orthopedic surgeries and health and social care costs.

A biomimetic/biomechanical approach has been developed in the design of a new modified dental material with bioactive ceramic hybrid material for biomechanical stimulation and potential improvement of the mineralization and ossification of the scaffold.

A polymer (pHEMA) filled with silica nanoparticles (4-6\% by volume) was chosen as biomimetic material.

This material swells (approximately 14\% linear) in the presence of an aqueous physiological solution (when in an aqueous biological environment), raising up to 50$30 \%$ by weight of water (depending on the nano-silicon load) of glass and soft rigid and rubber. The mechanical behavior of the proposed hybrid materials is comparable to that of the bone when vitreous and cartilage (ligaments) when it is rubber after swelling.

The bio-imitative properties of this very osteoconductive biomaterial have been used to develop a new bio-dental implant. The new concept is driven by the consideration that a bioactive interface between the implanted bone and the prosthetic device is generated when the material is able to stimulate the implant surrounding the bone in the physiological range of the strain for healthy bone remodeling and organized growth (50-3000).

The use of mechanically-compatible hybrid hydrogels as scaffolding materials is expected to increase prosthetic adaptation mechanisms that introduce active interfaces that improve the implant biomimetic while reproducing the biomechanical functions of cartilage and ligaments. The adaptive properties of biomimetic biomaterials (compatible with biomechanics and bioactive) are combined with new prostheses (Aversa et al., 2016a; 2016b; 2016c; 2016d).

Whenever we think of an organ of the human body we should look at it as an active component of the whole organism and not just as a separate organ. All matter has a certain life in the sense that it is born in a certain form and then it ages, undergoing permanent transformations that lead to its aging and changes of state, form and appearance. Organic living matter, unlike inorganic matter, is not composed of atomic chains that form molecules but is based on the living cell, the cell which is composed basically of atoms and molecules, including molecular chains, so unlike inorganic matter which is on essence structured from atoms and molecules, organic living matter is structured from living cells, which fulfill the fundamental roles of life as we know it today on our blue planet.

It is a great lack to look at a living organ belonging to an organism only from the anatomical point of view of the respective organ because each organ has several functionalities that it also performs in correlation with the other organs of the whole human body.

For example, the bone system has not only the apparently main function of supporting and defending the whole human body. Perhaps this role of dressing, supporting and protecting all the other organs is a basic one, but not the most essential one fulfilled by the bone system. The bone system has many roles to play in the human body, but its most important role is to support the circulatory system by creating fresh blood permanently even within the spinal cord, but also from young or adult bones, through the creation of all cells of the blood, of which there are mainly four types of cells, namely, the red cells that have the role of transporting and nourishing the whole body with oxygen, thus carrying out the oxygenation function of the whole organism up to the level of the cells, then the white cells of different types which have different roles in defending the body from various dangerous intruders, thirdly it is the platelet cells that will stop the bleeding that can start with the various wounds that are created in the life of the body and fourthly it is the stem cells essential for a rapid restoration of any type of cells depending on the organ that is affected. In general, stem cells that can also differentiate when they reach a particular organ are also produced within the bone marrow and are also transported by blood directly where it is necessary to repair a tissue belonging to a particular injured organ. For this reason, the human body is provided with a certain symbiosis between the emergency stem cell repair cells and the platelet cells that stop the rapid accidental bleeding produced in the body. This symbiosis occurs since their birth and for this reason, they are embedded together within placentae so 
that when circulating through the blood, they will be placentally coupled to both platelet and stem cells, being ready to act together as soon as possible an emergency, injury, or another extreme situation occurs within a point of the entire body. Platelet cells from the placental masses that enclose and transport them immediately stop bleeding to a wound produced and the stem cells immediately enter into action and begin tissue reconstruction at the affected site no matter what type of tissue or cells would be where the injury occurred because the initial stem cells created in the bone marrow are capable of developing and transforming into any type of human cell, adapting rapidly to any area or point of the human body, a huge advantage that these cells have compared to other cells that multiply in their workplace and by living permanently restoring the organ of which they belong, but which could not adapt to any other organ as the wonderful stem cells can do. So until now, four such basic products of bone marrow are known, as we have just shown, these are the main components of human blood, without which the blood that is restored permanently and regenerates in the bone marrow (so this fresh blood), would not it could exist and at that moment the body could no longer live.

Going back to the stem cells, it should be pointed out that they move through the blood within the body and if we want to collect them more easily so that they can be quickly injected into a certain place in the body in order to repair that area that was previously affected by something, then the easiest way to collect stem cells is to separate them together with platelets directly from the blood.

At this point, it is necessary to pause a little to highlight another essential role of the bone system within the body. The human body begins to age when it no longer produces enough fresh blood because of the lack of bone marrow that over time begins to become more less. The bone system together with the liver, lungs and kidneys, represents the main reservoir of water, minerals and blood, as well as the main storehouse of water, minerals and blood and thus indirectly and the main energy reservoir of the body. Specialized micron small cells, called mitochondria, have the primary role of producing energy for the body by creating energy ATP molecular chains, from water and air plus phosphorus, extracted from water and air from the body and from phosphorus from the bone reservoir, but then when the bone reservoir begins to lose from water and phosphorus reserves and the amount of energy raw material available to the mitochondria in the body decreases, leading to a decrease in the raw materials available for mitochondria and thus the energy production at the cellular level decreases and with it and the production of mitochondria and healthy new cells. Here it seems to be a vicious circle that is hard to stop, but in reality, the fault is genetically exclusive, because the bone system is genetically programmed to age, at one point the bones never can further produce marrow and blood and then only the ends of the bones help the spinal cord to produce the marrow and blood and at some point, they will not be able to do so and all the production of marrow and blood then remains in the spinal cord. If other organs can be permanently restored especially in the presence of fresh blood, the bones are sadly destined to age in several stages, genetically programmed. Although there is a large reserve of water and blood in the liver, kidneys and lungs, the basic reserve is that of bone, which has in its component water, minerals, phosphorus, blood, fat, able to stick and build the cell glue that is used in the cells but also between them, uniting the living cells to hold them together and in addition, only the bone reservoir can build the marrow of bone, spinal cord and blood, but also energy, basic energetic substances, to be donated to the mitochondria that will it then produces ATPs (energy molecular chains). If the bone system gets old and deteriorates over time, it is obvious that then the blood and energy will decrease and the water supply in the body will decrease and, at birth, it is $90 \%$, in a child, it becomes $80 \%$, in adolescents of about $70 \%$, in adults, it drops to $65 \%$ and in older people, it reaches $50 \%$ and below. Below $45 \%$ the water supply can no longer support the recovery of the blood in the required amount, nor of the ATP energy and the aging body will suffer from lack of energy, lack of oxygenation, lack of water and food, but also lack of extraction in time of normal toxins returned from the cells to the kidneys and liver also through the blood.

\section{Conclusion}

At least from this point of view, the restoration of the broken or accidentally broken bones should be done in such a way that it does not affect the bone tissues and their life and functions, so as not to prematurely age the human bone system, an essential system for sustaining the life and vitality of the body.

It is necessary to develop new biomaterial technologies to produce scaffolds and bone substitutes that could play a fundamental role in bone regeneration. Bone forms must exhibit specific intrinsic characteristics to function as a replacement of the actual bone, respecting biological, mechanical and geometric constraints.

These features include:

- Biological Requirements - Computerized schemes should allow cell adhesion and homogeneous distribution, growth of regenerative tissue and ensure the passage of nutrients and chemical signals. This achievement was achieved by controlling the scaffold porosity

- Mechanical requirements - Schemes must maintain mechanical and hardness properties that allow 
osteoblastic colonies to have physiologically and bio-actively controlled deformations. This was done by correctly modifying the composition of the ceramic-polymer composition (in our case, $10 \%$ by volume amorphous nano-silica)

The combination of clinical observation of traditional implantation behavior will be used to validate the bio-fidelity of FEM models, while the comparison of in vitro simulated in vitro growth of osteoblastic colonies would allow us to explore many new ideas in the design, design and fabrication of new structure nanostructured cellular cells with increased functionality and increased cellular interaction.

This proves to be particularly useful in the direct design and manufacture of the complex skeletal scaffold.

The new type of biomimetic implants can find applications in the knee, ankle, hip, shoulder and orthopedic column.

Another area of application of biomimetic schemes is surgical oncology to support and facilitate bone regeneration, resulting in massive losses due to primitive and metastatic tumor removal interventions.

The prosthetic system could allow for better functional recovery by promoting bone recreation to ensure good maintenance even if it will have an impact on the quality of life of the individual patient severely affected by the oncological pathology underlying it.

The biomimetic solution combining a metal support structure (to guarantee load resistance) an osteoinductive and biomechanically active bone (which promotes bone regeneration) finds replication in all surgical treatment areas involving bone removal and requires regenerative stimulation of the resected tissue. In fact, the concentrated bone marrow contains growth factors and mesenchymal stem cells that can specialize in bone cells, cartilage cells and tendons.

A biomimetic/biomechanical approach has been developed in the design of a new dental material modified with hybrid ceramic hybrid material for biomechanical stimulation and potential mineralization and ossification of the scaffold.

Polymer ( $\mathrm{pHEMA}$ ) filled with nano silica particles (4-6\% by volume) were chosen as biomimetic material. This material swells (approximately 14\% linear) in the presence of an aqueous physiological solution (when in an aqueous biological environment), raising up to 50$30 \%$ by weight of water (depending on the nano-silicon load) of glass and soft rigid and rubber.

The mechanical behavior of the proposed hybrid materials is comparable to that of the bone when it is glassy and cartilage (ligaments) when it is rubber after swelling.

The bio-imitative properties of this very osteoconductive biomaterial have been used to develop a new bioactive dental implant.
The new concept is driven by the fact that the bioactive interface of the scaffold between the implanted bone and the prosthetic device is generated when the material is able to stimulate the implant surrounding the stem to reshape the healthy bone and increase growth (50-3000).

The use of mechanically-compatible hybrid hydrogels as scaffolding materials is expected to increase prosthetic adaptation mechanisms that introduce active interfaces that improve the implant biomimetic while reproducing the biomechanical functions of cartilage and ligaments; (Adaptive properties of biomimetic biomaterials biomechanically and bioactive compatible of biomass, combined with new prostheses).

The present paper also proposes practical solutions to the need for bone repair, including dental, but also raises some questions regarding the importance of the bone system within the human body and how to preserve it for the longest period of time, questions to which some answers come even from the presented paper, but others will probably come in the future, because the problem of aging bones is obviously a genetic one and it cannot be solved instantly.

\section{Acknowledgement}

The Authors acknowledge Liquid Metals Technologies Inc, Ca USA that kindly supplies the samples for the characterization.

\section{Funding Information}

This research has been funded by Italian Ministry of University and Research project FIRB Future in Research 2008 project RBFR08T83J.

\section{Author's Contributions}

All the authors contributed equally to prepare, develop and carry out this manuscript.

\section{Ethics}

This article is original and contains unpublished material. Authors declare that are not ethical issues and no conflict of interest that may arise after the publication of this manuscript.

\section{References}

Adamopoulos, I.E., 2018. Inflammation in bone physiology and pathology. Curr. Opin. Rheumatol., 30: 59-64. DOI: 10.1097/BOR.0000000000000449

Ankrum, J. and J.M. Karp, 2010. Mesenchymal stem cell therapy: Two steps forward, one step back. Trends Mol. Med., 16: 203-209.

DOI: $10.1016 /$ j.molmed.2010.02.005 
Annunziata, M., R Aversa, A. Apicella, A. Annunziata and D. Apicella et al., 2006. In vitro biological response to a light-cured composite when used for cementation of composite inlays. Dental Mater., 22: 1081-1085. DOI: 10.1016/J.DENTAL.2005.08.009

Annunziata, M., L. Guida, L. Perillo, R. Aversa and I. Passaro et al., 2008. Biological response of human bone marrow stromal cells to sandblasted titanium nitride-coated implant surfaces. J. Mater. Sci. Mater. Med., 19: 3585-3591.

DOI: $10.1007 / \mathrm{s} 10856-008-3514-2$.

Apicella, A., B. Cappello, M.A. Del Nobile, M.I. La Rotonda and G. Mensitieri et al., 1993. Poly(ethylene oxide) (PEO) and different molecular weight PEO's blends monolithic devices for drug release. Biomaterials, 142: 83-90.

DOI: $10.1016 / 0142-9612(93) 90215-\mathrm{N}$

Apicella, A. and H. Hopfenberg, 1982. Water-swelling behavior of an ethylene-vinyl alcohol copolymer in the presence of sorbed sodium chloride. J. Applied Polymer Sci., 27: 1139-1148. DOI: 10.1002/app.1982.070270404

Apicella, D., R. Aversa, E. Ferro, D. Ianniello and A. Apicella, 2010. The importance of cortical bone orthotropicity, maximum stiffness direction and thickness on the reliability of mandible numerical models. J. Biomed. Mater. Res., 93: 150-163.

DOI: $10.1002 / \mathrm{jbm} . b .31569$

Apicella, D., M. Veltri, P. Balleri, A. Apicella and M. Ferrari, 2011. Influence of abutment material on the fracture strength and failure modes of abutmentfixture assemblies when loaded in a bio-faithful simulation. Clin. Oral Implants Res., 22: 182-188. DOI: $10.1111 / \mathrm{j} .1600-0501.2010 .01979 . x$

Apicella, D., R. Aversa, M. Tatullo, M. Simeone and S. Sayed et al. 2015. Direct restoration modalities of fractured central maxillary incisors: A multi-levels validated finite elements analysis with in vivo strain measurements. Dental Mater., 31: e289-e305.

DOI: $10.1016 /$ j.dental.2015.09.016

Aversa, R., D. Apicella, L. Perillo, R. Sorrentino and F. Zarone et al., 2009. Non-linear elastic threedimensional finite element analysis on the effect of endocrown material rigidity on alveolar bone remodeling process. Dental Mater., 25: 678-690: DOI: $10.1016 /$ j.dental.2008.10.015

Aversa, R., F.I.T. Petrescu, R.V. Petrescu and A. Apicella, 2016a. Biomimetic FEA bone modeling for customized hybrid biological prostheses development. Am. J. Applied Sci., 13: 1060-1067. DOI: 10.3844/ajassp.2016.1060.1067

Aversa, R., D. Parcesepe, R.V. Petrescu, G. Chen and F.I.T. Petrescu et al., 2016b. Glassy amorphous metal injection molded induced morphological defects. Am. J. Applied Sci., 13: 1476-1482. DOI: 10.3844 ajassp.2016.1476.1482
Aversa, R., R.V. Petrescu, F.I.T. Petrescu and A. Apicella, 2016c. Smart-factory: Optimization and process control of composite centrifuged pipes. Am. J. Applied Sci., 13: 1330-1341.

DOI: 10.3844/ajassp.2016.1330.1341

Aversa, R., F. Tamburrino, R.V. Petrescu, F.I.T. Petrescu and M. Artur et al., $2016 \mathrm{~d}$. Biomechanically inspired shape memory effect machines driven by muscle like acting NiTi alloys. Am. J. Applied Sci., 13: 1264-1271. DOI: 10.3844/ajassp.2016.1264.1271

Aversa, R., R.V.V. Petrescu, A. Apicella and F.I.T. Petrescu, 2017a. Nano-diamond hybrid materials for structural biomedical application. Am. J. Biochem. Biotechnol., 13: 34-41. DOI: 10.3844/ajbbsp.2017.34.41

Aversa, R., R.V.V. Petrescu, A. Apicella and F.I.T. Petrescu, 2017b. Modern transportation and photovoltaic energy for urban ecotourism. Transylvanian Rev. Admin. Sci. Special Issue. DOI: $10.24193 /$ tras.SI2017.1

Aversa, R., R.V.V. Petrescu, A. Apicella and F.I.T. Petrescu, 2019. A nanodiamond for structural biomimetic scaffolds. Eng. Rev., 39: 81-89. DOI: $10.30765 /$ er.39.1.9

Azi, M.L., A. Aprato, I. Santi, M. Kfuri and A. Masse et al., 2016. Autologous bone graft in the treatment of post-traumatic bone defects: A systematic review and meta-analysis. BMC Musculoskelet Disord, 17: 465-465. DOI: 10.1186/s12891-016-1312-4

Badziag, P., W.S. Verwoerd, W.P. Ellis and N.R. Greiner, 1990. Nanometre-sized diamonds are more stable than graphite. Nature, 343: 244-245. DOI: $10.1038 / 343244 a 0$

Barnard, A.S., S.P. Russo and I.K. Snook, 2003. Structural relaxation and relative stability of nanodiamond morphologies. Diamond Relat. Mater., 12: 1867-1872. DOI: 10.1016/S0925-9635(03)00275-9

Barnard, A.S. and M. Sternberg, 2007. Crystallinity and surface electrostatics of diamond nanocrystals. J. Mater. Chem., 17: 4811-4819.

DOI: $10.1039 / \mathrm{b} 710189 \mathrm{a}$

Beaupre, G.S. and W.C. Hayes, 1985. Finite element analysis of a three dimensional open-celled model for trabecular bone. J. Biomech. Eng., 107: 249-56. PMID: 4046566

Bonfield, W., M.D. Grynpas, A.E. Tully, J. Bowman and J. Abram, 1981. Hydroxyapatite reinforced polyethylene-a mechanically compatible implant material for bone replacement. Biomaterials, 2: $185-$ 186. DOI: 10.1016/0142-9612(81)90050-8

Braun, A.P. and H. Schulman, 1995. The multifunctional calcium/calmodulin-dependent protein kinase: From form to function. Annu. Rev. Physiol., 57: 417-445. DOI: 10.1146/annurev.ph.57.030195.002221 
Bueno, E.M. and J. Glowacki, 2009. Cell-free and cellbased approaches for bone regeneration. Nat. Rev. Rheumatol., 5: 685-697.

DOI: $10.1038 /$ nrrheum.2009.228

Chang, Y.R., J.H. Hsu, K. Chen and W. Fann, 2008. Mass production and dynamic imaging of fluorescent nanodiamonds. Nature Nanotech., 3: 284-288. DOI: 10.1038/nnano.2008.99

Cho, T.H., I.S. Kim, B. Lee, S.N. Park and J.H. Ko et al., 2017. Early and marked enhancement of new bone quality by alendronate-loaded collagen sponge combined with bone morphogenetic protein-2 at high dose: A long-term study in calvarial defects in a rat model. Tissue Eng. Part A, 23: 1343-1360. DOI: $10.1089 /$ ten.tea.2016.0557

Chow, E.K., X.Q. Zhang, M. Chen, R. Lam and E. Robinson et al., 2010. Nanodiamond therapeutic delivery agents mediate enhanced chemoresistant tumor treatment. Sci. Transl. Med., 3: 73ra21-73ra21. DOI: 10.1126/scitranslmed.3001713

Davis, P.A., S.J. Huang, L. Nicolais and L. Ambrosio, 1991. Modified PHEMA Hydrogels. In: High Performance Biomaterials, Szycher, M. (Ed.), Technonic, Lancaster, PA, USA, pp: 343-68.

Dimitriou, R., E. Jones, D. McGonagle and P.V. Giannoudis, 2011. Bone regeneration: Current concepts and future directions. BMC Med., 9: 66-66. DOI: $10.1186 / 1741-7015-9-66$

Filmon, R., F. Grizon, M.F. Baslie and D. Chappard, 2002. Effects of negatively charged groups (carboxymethyl) on the calcification of poly(2hydroxyethylmethacrylate). Biomaterials, 23: 3053-3059. DOI: 10.1016/S0142-9612(02)00069-8

Frost, H.M., 1964. Mathematical elements of lamellar bone remodeling. Charles C Thomas, Springfield.

Frost, H.M., 1990. Structural Adaptations to Mechanical Usage (SATMU). 2. Redifining Wolff's law: The bone remodelling problem. Anat. Rec., 226: 414-422. DOI: $10.1002 /$ ar. 1092260403

Frost, H.M., 2003. Update of bone physiology and Wolff's law for clinicians. Angle Orthod., 74: 3-15. DOI: 10.1051 /orthodfr/200374015

Frost, H.M., 1994. Wolff's law and bone's structural adaptations to mechanical usage: An overview for clinicians. Angle Orthod., 64: 175-188.

Geiger, M., R.H. Li and W. Friess, 2003. Collagen sponges for bone regeneration with rhBMP-2. Adv. Drug Deliv. Rev., 55: 1613-1629. DOI: 10.1016/j.addr.2003.08.010

Gerber, H.P., T.H. Vu, A.M. Ryan, J. Kowalski and Z. Werb et al., 1999. VEGF couples hypertrophic cartilage remodeling, ossification and angiogenesis during endochondral bone formation. Nat. Med., 5: 623-623.

Glowacki, J., 1998. Angiogenesis in fracture repair. Clin. Orthop. Relat. Res., 355: S82-S89.
Gorustovich, A.A., J.A. Roether and A.R. Boccaccini, 2010. Effect of bioactive glasses on angiogenesis: A review of in vitro and in vivo evidences. Tissue Eng. Part B Rev., 16: 199-207. DOI: $10.1089 /$ ten.TEB.2009.0416

Gramanzini, M., S. Gargiulo, F. Zarone, R. Megna and A. Apicella et al., 2016. Combined microcomputed tomography, biomechanical and histomorphometric analysis of the peri-implant bone: A pilot study in minipig model. Dental Mater., 32: 794-806.

DOI: 10.1016/j.dental.2016.03.025

Greiner, N.R., D.S. Phillips, J.D. Johnson and F. Volk, 1988. Diamonds in detonation soot. Nature, 333: 440-442. DOI: $10.1038 / 333440 \mathrm{a} 0$

Ha, W.H., H.S. Seong, N.R. Choi, B.S. Park and Y.D. Kim, 2017. Recombinant human bone morphogenic protein-2 induces the differentiation and mineralization of osteoblastic cells under hypoxic conditions via activation of protein kinase $\mathrm{D}$ and p38 mitogen-activated protein kinase signaling pathways. Tissue Eng. Regen Med., 14: 433-441.

Halpin, J.C. and J.L. Kardos, 1976. Halpin-Tsai equations: A review. Polymer Eng. Sci., 16: 344-352.

Hench, L.L. and J.M. Polak, 2002. Third-generation biomedical materials. Science, 295: 1014-1017. DOI: $10.1126 /$ science. 1067404

Hench, L.L. and I. Thompson, 2010. Twenty-first century challenges for biomaterials. J. Royal Society Interface, 7: S379-S391. DOI: 10.1098/rsif.2010.0151.focus

Hench, L.L. and J. Wilson, 1993. An introduction to bioceramics. World Sci., 1: 396-396. DOI: $10.1142 / 2028$

Hogan, P.G., L. Chen, J. Nardone and A. Rao, 2003. Transcriptional regulation by calcium, calcineurin and NFAT. Gene Dev., 17: 2205-2232.

Holley, R.H., H.B. Hopfenberg and V. Stannett, 1970. Anomalous transport of hydrocarbons in polystyrene. Polymer Eng. Sci., 10: 376-382. DOI: 10.1002/pen.760100612

Hoppe, A., N.S. Güldal and A.R. Boccaccini, 2011. A review of the biological response to ionic dissolution products from bioactive glasses and glass-ceramics. Biomaterials, 32: 2757-2774. DOI: $10.1016 /$ j.biomaterials.2011.01.004

Huiskes, R., H. Weinans, H.J. Grootenboer, M. Dalstra and B. Fudula et al., 1987. Adaptive bone remodeling theory applied to prosthetic-design analysis. J. Biomech., 20: 1135-1150.

Hutmacher, D.W., 2000. Scaffolds in tissue engineering bone and cartilage. Biomaterials, 21: 2529-2543. DOI: 10.1016/S0142-9612(00)00121-6

Jarre, G., Y.J. Liang, P. Betz, D. Lang and A. Krueger, 2011. Playing the surface game-Diels-Alder reactions on diamond nanoparticles. Chem. Commun., 47: 544-546. DOI: 10.1039/C0CC02931A 
Jones, J.R. and A.G. Clare, 2012. Bio-Glasses: An Introduction. 1st Edn., Wiley, Chichester, ISBN-10: 1118346475 , pp: 320.

Julien, M., D. Magne, M. Masson, M. Rolli-Derkinderen and O. Chassande et al., 2007. Phosphate stimulates matrix Gla protein expression in chondrocytes through the extracellular signal regulated kinase signaling pathway. Endocrinology, 148: 530-537. DOI: $10.1210 /$ en.2006-0763

Kabra, B., S.H. Gehrke, S.T. Hwang and W. Ritschel, 1991. Modification of the dynamic swelling behaviour of pHEMA. J. Applied Polym. Sci., 42: 2409-2416. DOI: 10.1002/app.1991.070420906

Kahl, C.R. and A.R. Means, 2003. Regulation of cell cycle progression by calcium/calmodulin-dependent pathways. Endocr. Rev., 24: 719-736.

Karageorgiou, V. and D. Kaplan, 2005. Porosity of 3D biomaterial scaffolds and osteogenesis. Biomaterials, 26: 5474-5491.

DOI: 10.1016/j.biomaterials.2005.02.002

Kim, R.Y., Y. Seong, T.H. Cho, B. Lee and I.S. Kim et al., 2018. Local administration of the Nuclear Factor of Activated T cells (NFAT) cl inhibitor to suppress early resorption and inflammation induced by bone morphogenetic protein $\square$ 2. J. Biomed. Mater. Res. A, 106: 1299-1310.

Kim, S.K., T.H. Cho, J.J. Han, I.S. Kim and Y. Park et al., 2016. Comparative study of BMP-2 alone and combined with VEGF carried by hydrogel for maxillary alveolar bone regeneration. Tissue Eng. Regen Med., 13: 171-181.

Kim, J., H.J. Yang, T.H. Cho, S.E. Lee and Y.D. Park et al., 2013. Enhanced regeneration of rabbit mandibular defects through a combined treatment of electrical stimulation and rhBMP-2 application. Med. Biol. Eng. Comput., 51: 1339-1348.

Kim, J.H., T.H. Lee, Y.M. Song, I.S. Kim and T.H. Cho et al., 2011. An implantable electrical bioreactor for enhancement of cell viability. Proceedings of the Annual International Conference of the IEEE Engineering in Medicine and Biology Society, Aug. 30-Sept. 3, IEEE Xplore Press, Boston, MA, USA, pp: 3601-3604. DOI: 10.1109/IEMBS.2011.6090603

Kim, I.S., E.N. Lee, T.H. Cho, Y.M. Song and S.J. Hwang et al., 2010. Promising efficacy of Escherichia coli recombinant human bone morphogenetic protein-2 in collagen sponge for ectopic and orthotopic bone formation and comparison with mammalian cell recombinant human bone morphogenetic protein-2. Tissue Eng. Part A, 17: 337-348.

Kim, H.W., J.C. Knowles and H.E. Kim, 2004. Development of hydroxyapatite bone scaffold for controlled drug release via poly( $\square$-caprolactone) and hydroxyapatite hybrid coatings. J. Biomed. Mater. Res. Part B: Applied Biomater., 70: 240-249. DOI: $10.1002 / \mathrm{jbm} . b .30038$
Krueger, A., J. Stegk, Y.J. Liang, L. Lu and G.B. Jarre, 2008. Nanodiamond: Simple and efficient functionalization of detonation diamond. $\mathrm{E}$ Langmuir, 24: 4200-4204. DOI: 10.1021/la703482v

Krueger, A., Y.J. Liang, G. Jarre and J. Stegk, 2006, Surface functionalisation of detonation diamond suitable for biological applications. J. Mater. Chem., 16: 2322-2328. DOI: 10.1039/B601325B

Lai, L. and A.S. Barnard, 2011a. Modeling the thermostability of surface functionalisation by oxygen, hydroxyl and water on nanodiamonds. Nanoscale, 3: 2566-2575. DOI: 10.1039/clnr10108k

Lai, L. and A.S. Barnard, 2011b. Stability of nanodiamond surfaces exposed to N, NH and NH2. J. Phys. Chem. C, 115: 6218-6228. DOI: 10.1021/jp1111026

Lee, S.W., F. Fallegger, B.D.F. Casse and S.I. Fried, 2016. Implantable microcoils for intracortical magnetic stimulation. Sci. Adv., 2: e1600889-e1600889.

Lim, J., Z.R.M. Razi, J.X. Law, A.M. Nawi and R.B.H. Idrus et al., 2018. Mesenchymal stromal cells from the maternal segment of human umbilical cord is ideal for bone regeneration in allogenic setting. Tissue Eng. Regen Med., 15: 75-87.

Mano, J.F., R.A. Sousa, L.F. Boesel, N.M. Neves and R.L. Reis, 2004. Bioinert, biodegradable and injectable polymeric matrix composites for hard tissue replacement: State of the art and recent developments. Compos. Sci. Technol., 64: 789-817. DOI: 10.1016/j.compscitech.2003.09.001

Marrelli, M., G. Falisi, A. Apicella, D. Apicella and M. Amantea et al., 2015. Behaviour of dental pulp stem cells on different types of innovative mesoporous and nanoporous silicon scaffolds with different functionalizations of the surfaces. J. Biol. Regulators Homeostatic Agents, 9: 991-997. PMID: 26753666

Martin, R.B., D.B. Burr and N.A. Sharkey, 1998. Skeletal Tissue Mechanics. 1st Edn., Springer, New York, ISBN-10: 0387984747, pp: 392.

Mesfin, A., J.M. Buchowski, L.P. Zebala, W.R. Bakhsh and A.B. Aronson et al., 2013. High-dose rhBMP-2 for adults: major and minor complications: A study of spine cases. J. Bone Joint Surg. Am., 95: 1546-1553.

Montheard, J.P., M. Chatzopoulos and D. Chappard, 1992. 2-hydroxyethylmethacrylate HEMA; chemical properties and applications in biomedical fields. J. Macromol. Sci. Macromol. Rev., 32: 1-34.

DOI: $10.1080 / 15321799208018377$

Morales-Hernandez, D.G., D.C. Genetos, D.M. Working, K.C. Murphy and J.K. Leich, 2012. Ceramic identity contributes to mechanical properties and osteoblast behavior on macroporous composite scaffolds. J. Funct. Biomat., 23: 382-397. DOI: $10.3390 / \mathrm{jfb} 3020382$ 
Mouriño, V., J.P. Cattalini and A.R. Boccaccini, 2012. Metallic ions as therapeutic agents in tissue engineering scaffolds: An overview of their biological applications and strategies for new developments. J. Royal Society Interface, 9: 401-419. DOI: $10.1098 /$ rsif.2011.0611

Nicolais, L., A. Apicella and C. de Notaristefano, 1984. Time-temperature superposition of n-hexane sorption in polystyrene. J. Membrane Sci., 18: 187-196. DOI: $10.1016 / \mathrm{S} 0376-7388(00) 85033-4$

Oliveira, S.M., R.A. Ringshia, R.Z. Legeros, E. Clark and M.J. Yost et al., 2010. An improved 711 collagen scaffold for skeletal regeneration. J. Biomed. Mater. Res. A, 94A: 371-379.

Osswald, S., G. Yushin, V. Mochalin, S.O. Kucheyev and Y. Gogotsi, 2006. Control of sp2/sp3 carbon ratio and surface chemistry of nanodiamond powders by selective oxidation in air. J. Am. Chem. Soc., 128: 11635-11642. DOI: 10.1021/ja063303n

Ozawa, M., M. Inaguma, M. Takahashi, F. Kataoka and A. Krüger et al., 2007. Preparation and behavior of brownish, clear nanodiamond colloids. Adv. Mater., 19: 1201-1206. DOI: 10.1002/adma.200601452

Parfitt, A.M., 1983. The Physiological and Clinical Significance of Bone Histomorphometric Data. In: Bone Histomorphometry: Techniques and Interpretation, Recker, R.R. (Ed.), CRC Press, Boca Raton, pp: 143-223.

Parfitt, A.M., 1994. Osteonal and hemi-osteonal remodeling: The spatial and temporal framework for signal traffic in adult human bone. J. Cell Biochem., 55: 273-286. DOI: 10.1002/jcb.240550303

Peluso, G., O. Petillo, J.M. Anderson, M. Ambrosio and L. Nicolais et al., 1997. The differential effects of poly(2-hydroxyethylmethacrylate) and poly(2hydroxyethylmethacrylate)/poly(caprolactone) polymers on cell proliferation and collagen synthesis by human lung fibroblasts. J. Biomed. Mater. Res., 34: 327-336. DOI: $10.1002 /(\mathrm{SICI}) 1097-$ 4636(19970305)34:3<327::AID-JBM7>3.0.CO;2-M

Perillo, L., R. Sorrentino, D. Apicella, A. Quaranta and E.D. Gherlone et al., 2010. Nonlinear visco-elastic finite element analysis of porcelain veneers: A submodelling approach to strain and stress distributions in adhesive and resin cement. J. Adhesive Dentistry, 12: 403-413.

Petrescu, F.I.T. and K.J. Calautit, 2016a. About nano fusion and dynamic fusion. Am. J. Applied Sci., 13: 261-266.

Petrescu, F.I.T. and K.J. Calautit, 2016b. About the light dimensions. Am. J. Applied Sci., 13: 321-325.

Petrescu, F.L., E. Buzea, L. Nănuț, M. Neacşa and C. Nan, 2015. The role of antioxidants in slowing aging of skin in a human, Analele Univers. Craiova Biologie Horticultura Tehn. Prel. Prod. Agr. Ing. Med., 20: 567-574.
Petrescu, F.I.T., A. Apicella, R. Aversa, R.V. Petrescu and J.K. Calautit et al., 2016a. Something about the mechanical moment of inertia. Am. J. Applied Sci., 13: 1085-1090.

Petrescu, R.V., R. Aversa, A. Apicella, S. Li and G. Chen et al., 2016b. Something about electron dimension. Am. J. Applied Sci., 13: 1272-1276.

Petrescu, R.V., R. Aversa, A. Apicella, S. Li and F.I.T. Petrescu, 2016c. Ecosphere protection through green energy. Am. J. Applied Sci., 13: 1027-1032.

Petrescu, F.I.T., A. Apicella, R.V. Petrescu, S.P. Kozaitis and R.B. Bucinell et al., 2016d. Environmental protection through nuclear energy. Am. J. Applied Sci., 13: 941-946.

Petrescu, F.I.T. and R.V. Petrescu, 2017. The computer algorithm for machine equations of classical distribution. J. Mater. Eng. Struct., 4: 193-209.

Petrescu, F.I.T. and R.V. Petrescu, 2018. Inverse kinematics to a Stewart platform. J. Mater. Eng. Struct., 5: 111-122.

Petrescu, F.I.T. and R.V.V. Petrescu, 2019. Nuclear hydrogen structure and dimensions. Int. J. Hydrogen Energy, 44: 10833-10837.

Petrescu, F.I.T., 2019. About the nuclear particles' structure and dimensions. Comp. Part. Mech., 6: 191-194.

Pinto, M.C.X., A.H. Kihara, V.A. Goulart, F.M. Tonelli and K.N. Gomes et al., 2015. Calcium signaling and cell proliferation. Cell Signal, 27: 2139-2149.

Rattay, F., 1989. Analysis of models for extracellular fiber stimulation. IEEE Trans. Biomed. Eng., 36: 676-682.

Reilly, D. and A.H. Burstein, 1974. The mechanical properties of cortical bone. J. Bone Joint Surgery, 56: 1001-1021

Reilly, D.T. and A.H. Burnestain, 1975. The elastic and ultimate properties of compact bone tissue. J. Biomechan., 8: 393-405. DOI: 10.1016/0021-9290(75)90075-5

Schiraldi, C., A. D'Agostino, A. Oliva, F. Flamma and A. De Rosa et al., 2004. Development of hybridmaterials based on hydroxyethylmethacrylate as supports for improving cell adhesion and proliferation. Biomaterials, 25: 3645-3653. DOI: 10.1016/j.biomaterials.2003.10.059

Schrand, A.M., J. Johnson, L. Dai and E. Ōsawa, 2009a. Cytotoxicity and Genotoxicity of Carbon Nanomaterials. In: Safety of Nanoparticles: From Manufacturing to Medical Applications, Nanostructure Science and Technology, Webster, T.J. (Ed.), ISSN-10: 1571-5744, pp: 159-187.

Schrand, A.M., S.A.C. Hens and O.A. Shenderova, 2009b. Nanodiamond particles: Properties and perspectives for bioapplications. Crit. Rev. Solid State Mater. Sci., 34: 18-74. DOI: 10.1080/10408430902831987 
Schwartz-Dabney, C.L. and P.C. Dechow, 2003. Variation in cortical material properties throughout the human dentate mandible. Am. J. Phys. Anthropol., 120: 252-277. DOI: 10.1002/ajpa.10121

Shenderova, O., A. Koscheev, N. Zaripov, I. Petrov and Y. Skryabin et al., 2011. Surface chemistry and properties of ozone-purified detonation nanodiamonds. J. Phys. Chem. C, 115: 9827-9837. DOI: $10.1021 /$ jp1 102466

Shields, L.B.E., G.H. Raque, S.D. Glassman, M. Campbell and T. Vitaz et al., 2006. Adverse effects associated with high-dose recombinant human bone morphogenetic protein-2 use in anterior cervical spine fusion. Spine, 31: 542-547.

Song, W.Y., G.M. Liu, J. Li and Y.G. Luo, 2016. Bone morphogenetic protein-2 sustained delivery by hydrogels with microspheres repairs rabbit mandibular defects. Tissue Eng. Regen Med., 13: 750-761.

Sorrentino, R., D. Apicella, C. Riccio, E.D. Gherlone and F. Zarone et al., 2009. Nonlinear visco-elastic finite element analysis of different porcelain veneers configuration. J. Biomed. Mater. Res., 91: 727-736. DOI: $10.1002 / \mathrm{jbm} . b .31449$

Sorrentino, R., R. Aversa, V. Ferro, T. Auriemma and F. Zarone et al., 2007. Three-dimensional finite element analysis of strain and stress distributions in endodontically treated maxillary central incisors restored with different post, core and crown materials. Dent Mater., 23: 983-993. DOI: $10.1016 /$ j.dental.2006.08.006

Stuehr, D.J. and M.M. Haque, 2019. Nitric oxide synthase enzymology in the 20 years after the Nobel Prize. Br. J. Pharmacol., 176: 177-188.

Syed, J., A.A.L. Dharrab, M.S. Zafa, E. Khand and R. Aversa et al., 2017. Influence of curing light type and staining medium on the discoloring stability of dental restorative composite. Am. J. Biochem. Biotechnol. 13: 42-50. DOI: 10.3844 /ajbbsp.2017.42.50

Taylor, D., J.G. Hazenberg and T.C. Lee, 2007. Living with cracks: Damage and repair in human bone. Nat. Mater., 6: 263-268. DOI: 10.1038/nmat 1866

Töyräsa, J., T. Lyyra-Laitinena, M. Niinimäkib, R. Lindgrenc and M.T. Nieminenb et al., 2001. Estimation of the Young's modulus of articular cartilage using an arthroscopic indentation instrument and ultrasonic measurement of tissue thickness. J. Biomechan., 34: 251-256. DOI: 10.1016/S0021-9290(00)00189-5

Urist, M.R. and B.S. Strates, 1971. Bone morphogenetic protein. J. Dent. Res., 50: 1392-1406.

Viecelli, J.A., S. Bastea, J.N. Glosli and F.H. Ree, 2001. Phase transformations of nanometer size carbon particles in shocked hydrocarbons and explosives. J. Chem. Phys., 115: 2730-2736.

DOI: $10.1063 / 1.1386418$
Wheeler, D.G., C.F. Barrett, R.D. Groth, P. Safa and R.W. Tsien, 2008. CaMKII locally encodes L-type channel activity to signal to nuclear CREB in excitation-transcription coupling. J. Cell Biol., 183: 849-863.

Wiltfang, J., M. Rohnen, J.H. Egberts, U. Lützen and H. Wieker et al., 2016. Man as a living bioreactor: Prefabrication of a custom vascularized bone graft in the gastrocolic omentum. Tissue Eng. Part C Meth., 22: 740-746.

Winslow, M.M., M. Pan, M. Starbuck, E.M. Gallo and L. Deng et al., 2006. Calcineurin/NFAT signaling in osteoblasts regulates bone mass. Dev. Cell, 10: 771-782.

Wolff, J., 1892. Das Gesetz der Transformation der Knochen. A Hirschwald, Berlin.

Xiao, J., G. Ouyang, P. Liu, C.X. Wang and G.W. Yang, 2014. Reversible nanodiamond-carbon onion phase transformations. Nano Lett., 14: 3645-3652. DOI: $10.1021 / \mathrm{nl} 5014234$

Yoon, Y., I.U. Khan, K.U. Choi, T. Jung and K. Jo et al., 2018. Different bone healing effects of undifferentiated and osteogenic differentiated mesenchymal stromal cell sheets in canine radial fracture model. Tissue Eng. Regen Med., 15: 115-124.

Yuan, Y., X. Wang, G. Jia, J.H. Liu and T. Wang et al., 2010. Pulmonary toxicity and translocation of nanodiamonds in mice. Diamond Relat. Mater., 19: 291-299. DOI: 10.1016/j.diamond.2009.11.022

Zara, J.N., R.K. Siu, X. Zhang, J. Shen and R. Ngo et al., 2011. High doses of bone morphogenetic protein 2 induce structurally abnormal bone and inflammation in vivo. Tissue Eng. Part A, 17: 1389-1399.

Zhang, Q., V.N. Mochalin, I. Neitzel, I.Y. Knoke and J. Han et al. 2011. Fluorescent PLLA-nanodiamond composites for bone tissue engineering. Biomaterials, 32: 87-94.

DOI: 10.1016/j.biomaterials.2010.08.090 